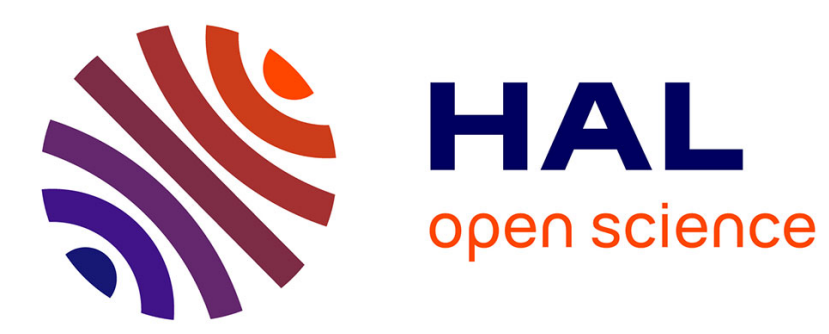

\title{
INERTIAL MANIFOLDS FOR THE HYPERVISCOUS NAVIER-STOKES EQUATIONS
}

\author{
Ciprian G Gal, Yanqiu Guo
}

\section{To cite this version:}

Ciprian G Gal, Yanqiu Guo. INERTIAL MANIFOLDS FOR THE HYPERVISCOUS NAVIERSTOKES EQUATIONS. 2017. hal-01490190v2

\section{HAL Id: hal-01490190 \\ https://hal.science/hal-01490190v2}

Preprint submitted on 7 May 2018

HAL is a multi-disciplinary open access archive for the deposit and dissemination of scientific research documents, whether they are published or not. The documents may come from teaching and research institutions in France or abroad, or from public or private research centers.
L'archive ouverte pluridisciplinaire HAL, est destinée au dépôt et à la diffusion de documents scientifiques de niveau recherche, publiés ou non, émanant des établissements d'enseignement et de recherche français ou étrangers, des laboratoires publics ou privés. 


\title{
INERTIAL MANIFOLDS FOR THE HYPERVISCOUS NAVIER-STOKES EQUATIONS
}

\author{
CIPRIAN G. GAL AND YANQIU GUO
}

\begin{abstract}
We prove the existence of inertial manifolds for the incompressible hyperviscous Navier-Stokes equations on the two or three-dimensional torus:$$
\left\{\begin{array}{l}
u_{t}+\nu(-\Delta)^{\beta} u+(u \cdot \nabla) u+\nabla p=f, \quad(t, x) \in \mathbb{R}_{+} \times \mathbb{T}^{d}, \\
\operatorname{div} u=0,
\end{array}\right.
$$

where $d=2$ or 3 and $\beta \geq 3 / 2$. Since the spectral gap condition is not necessarily satisfied for the aforementioned problem in three dimensions, we employ the spatial averaging method introduced by Mallet-Paret and Sell in [26].
\end{abstract}

\section{INTRODUCTION}

Let us consider the following hyperviscous version of the Navier-Stokes equations of incompressible fluid flow on a torus,

$$
\left\{\begin{array}{l}
u_{t}+\nu(-\Delta)^{\beta} u+(u \cdot \nabla) u+\nabla p=f, \quad(t, x) \in \mathbb{R}_{+} \times \mathbb{T}^{d} \\
\operatorname{div} u=0,\left.\quad u\right|_{t=0}=u_{0}
\end{array}\right.
$$

where $\beta \geq d / 4+1 / 2$ for the spatial dimension $d \geq 3$, and $\beta \geq 1$ for $d=2$. Here, $\mathbb{T}^{d}=[-\pi, \pi]^{d}$ is endowed with the periodic boundary condition. It is well-known that the regularized system (1.1) is globally well-posed in the $L^{2}$ space (see, e.g., [8]). It has been used, among others, by numerical analysts as a substitute model for the standard case $\beta=1$ and plays a key role in understanding turbulent phenomena in science (cf. Avrin [2], Borue and Orszag [4], Basdevant et. al. [6], Browning and Kreiss [7], Cerruto et. al. [10], Fornberg [20] and McWilliams [27]). Besides, the system (1.1) has some physical meaning (see again [10]). Given the nonlinear nature of turbulent incompressible viscous flows and the ensuing multiscale interactions, the direct numerical simulation of the Navier-Stokes equations is still presently lacking apart from some investigations performed on regularized systems which still retain the basic nonlinear structure and the essential features of the full hydrodynamic Navier-Stokes equations (cf. Holst et. al. [22], Gal and Medjo [21]). Among such regularized models, it is worth noting the following globally well-posed systems in three dimensions: the Leray- $\alpha$ model (cf. Cheskidov et. al. [11]), the simplified Bardina model (cf. Cao et. al. [9]), the Navier-Stokes- $\alpha$ equations (cf. Foias et. al. [16]) and the Navier-Stokes-Voight equations (cf. Kalantarov and Titi [23, Coti-Zelati and Gal [14]). One advantage of using the hyperviscous Navier-Stokes equations (1.1) with $\beta \geq 5 / 4$ in the three-dimensional domain is that the system only modifies the spectral distribution of

Date: May 6, 2018.

Key words and phrases. inertial manifold, Navier-Stokes equations, global attractor, hyperviscosity. 
energy, and the global well-posedness (i.e., existence, uniqueness and stability with respect to the initial data) of the solutions can be rigorously proven unlike for the three-dimensional Navier-Stokes equations (see [2, 8]). On the other hand, (1.1) has also been proposed and investigated for the purpose of direct numerical simulations of turbulent incompressible viscous flows, although orders of dissipation $\beta \geq 2$ have typically been used (see again [7, 10, 20, 27]). The existence of finite-dimensional global attractors for the regularized family (1.1) can been proven by employing standard theory of attractors. For the sake of completeness, we provide a brief proof of the global well-posedness and existence of global attractors of (1.1) in the appendix.

Furthermore, by directly verifying a spectral gap condition it was shown in [33] that the hyperviscous Navier-Stokes equations 1.1 possess an inertial manifold in $L^{2}$ provided $\beta>d$ for any spatial dimension $d \geq 2$. Exploiting the same strategy and a more refined analysis, the existence of inertial manifolds for (1.1) with $\beta>5 / 2$ in three-dimensional domains was established in [3] (the results in [3] actually hold for a general family of hyperviscous operators). The purpose of this manuscript is to prove the existence of an inertial manifold for (1.1) with $\beta \geq 3 / 2$ in two or three dimensions when the spectral gap condition is not necessarily fulfilled. The motivation for this line of research is the longstanding open problem concerning the existence of inertial manifolds for the Navier-Stokes equation $(\beta=1)$.

We recall that the existence of an inertial manifold guarantees that, in the long-term, the system resembles a finite-dimensional system of ordinary differential equations, which describes the limit dynamics of the original system as time goes to infinity (see, e.g. [18, 35]). Indeed, spectral gap conditions have been widely used in the literature to establish the existence of inertial manifolds for many dissipative evolution equations (cf. [1, 13, 17, 18, 19, 32, 34]). However, for a system that lacks the spectral gap condition, in their pioneering work [26, Mallet-Paret and Sell have introduced the so-called spatial averaging method to prove the existence of inertial manifolds for a three-dimensional reaction-diffusion equation. This technique was further simplified by Zelik [35, and extended by Kostianko and Zelik to the three-dimensional Cahn-Hilliard equation [25], and by Kostianko to the three-dimensional modified Leray- $\alpha$ model [24].

In the present work, we employ the spatial averaging method to establish the existence of inertial manifolds for the system (1.1) in the hyperdissipation range $\beta \geq 3 / 2$ in three dimensions when the spectral gap condition fails, whereas in two dimensions an arbitrarily large spectral gap is available. We draw techniques from the contributions [18, 24, 25, 26, 35. In particular, we would like to mention the article [25] by Kostianko and Zelik in which they studied the existence of inertial manifolds for a model problem that can be associated with the Cahn-Hilliard equation subject to periodic boundary conditions on $\mathbb{T}^{3}$. The model problem in [25] reads

$$
u_{t}+A^{2} u+A F(u)=0,\left.\quad u\right|_{t=0}=u_{0},
$$

where $A$ is a positive self-adjoint operator in some Hilbert space $H$, with compact inverse. The Cahn-Hilliard equation on $\mathbb{T}^{3}$ is an example of $(1.2)$ when $A=-\Delta$ with periodic boundary conditions on $\mathbb{T}^{3}$ and $F(u)=u^{3}-u$. It is well known that the Cahn-Hilliard equation can be also obtained as the conserved gradient flow in $H^{-1}$ (i.e., the dual of a proper subspace of $H^{1}$ ) associated with the Fréchet derivative of some free energy functional. 
Indeed, the Cahn-Hilliard equation is naturally dissipative in $H^{-1}$ in the sense that the solution semigroup is well-defined in $H^{-1}$, possessing an absorbing ball in $H^{-1}$. Based on this interpretation, one may then employ analytical techniques that are similar to ones for the reaction-diffusion equation $u_{t}+A u+F(u)=0$. Roughly speaking, this is also the approach used in [25] where in order to verify the existence of an inertial manifold for the Cahn-Hilliard equation, one constructs invariant cones in $H^{-1}$ and adopts the spatial averaging method that was initially developed for the $3 \mathrm{D}$ reaction-diffusion equation in [26].

Inspired by [25, 26, 35], we study an abstract model in some Hilbert space $H$,

$$
u_{t}+A^{\beta} u+A^{1 / 2} F(u)=f,\left.\quad u\right|_{t=0}=u_{0},
$$

for $\beta \geq 3 / 2$. Notice that the hyperviscous Navier-Stokes equations 1 with $\nu=1$ coincides with the model problem 1.3 when $A=-\Delta$ and $F(u)=A^{-1 / 2} B(u, u)$, where $B(u, v)=P_{\sigma}((u \cdot \nabla) v)$ is the bilinear form associated with the advection term in (1.1). Here $P_{\sigma}$ is the Helmholtz-Leray orthogonal projection operator. Then one needs to extend the aforementioned invariant cone and spatial averaging method to our model $(1.3)$ for establishing the existence of a Lipschitz inertial manifold. Our argument also relies on number theoretic results from [29] by Richards, and from [26] by Mallet-Paret and Sell. Specifically, we explicitly use the fact that the size of the gap among certain quadratic forms of integers is logarithmic growing (See Theorem 4.3). The most technical and essential content of this paper is contained in the proof of Theorem 2.4.

This manuscript is divided into sections as follows. Section 2 is devoted to a treatment of the model problem $(1.3$ by finding sufficient conditions that guarantee the existence of an inertial manifold. In particular, in Subsection 2.1, we state a well-known optimal spectral gap condition which immediately implies the existence of inertial manifolds for (1.3). Next, in Subsection 2.2 we adapt the method of spatial averaging developed in [26] and further exploited by [25], to treat the critical case $\beta=3 / 2$ of $(1.3)$, when the spectral gap condition is violated. Section 3 is devoted to an application of the theory established on the abstract model (1.3) to the hyperviscous Navier-Stokes equations (1.1). Specifically, in Subsection 3.1 we briefly verify the existence of a compact global attractor for (1.1) with $\beta \geq 3 / 2$ by showing the existence of an absorbing ball in $H^{4}$. Then, in Subsection 3.2 . by adopting the technique in [24, we modify (1.1) outside of this absorbing ball in such a way that the new equation possesses an inertial manifold. As usual, this inertial manifold will still be invariant with respect to the solution semigroup associated with (1.1) at least in the neighborhood of the global attractor and therefore will contain all of its non-trivial dynamics. All the corresponding conditions of our abstract theorem are verified directly in Subsection 3.3. The appendix contains some supporting material from number theory (see Appendix 4.1), and the proof of a standard result which says the cone invariance property and the decay property together imply the existence of inertial manifolds for the model problem (1.3) (see Appendix 4.2). For the sake of completeness, in the appendix we also provide the verification of the strong cone property for 1.3 when the optimal spectral gap condition is fulfilled (see Appendix 4.3), as well as a brief proof of the global wellposedness of weak solutions and the existence of the global attractor for the hyperviscous Navier-Stokes equations (1.1) for $\beta \geq 5 / 4$ in three dimensions (see Appendix 4.4). 


\section{THE ABSTRACT MODEL}

First, let $H$ be a Hilbert space. We study the following abstract model in $H$,

$$
u_{t}+A^{\beta} u+A^{1 / 2} F(u)=f,\left.\quad u\right|_{t=0}=u_{0},
$$

for $\beta \geq 3 / 2$. Here $F$ is an operator mapping from $H$ to $H$, and $A: \mathcal{D}(A) \rightarrow H$ is a linear self-adjoint positive operator with compact inverse. The operator $A$ possesses the complete orthonormal system of eigenvectors $\left\{e_{j}\right\}_{j=1}^{\infty}$ in $H$ which corresponds to eigenvalues $\lambda_{j}$ such that $\lambda_{j} \rightarrow \infty$ as $j \rightarrow \infty$ and

$$
A e_{j}=\lambda_{j} e_{j}, \quad 0<\lambda_{1} \leq \lambda_{2} \leq \lambda_{3} \leq \cdots
$$

Throughout, we denote by $|\cdot|$ the norm of $H$ and $(\cdot, \cdot)$ denotes the inner product in $H$. For $N \in \mathbb{N}$, let us now define the following projection operators

$$
P_{N} v=\sum_{j=1}^{N} v_{j} e_{j}, Q_{N} v=\sum_{j=N+1}^{\infty} v_{j} e_{j}
$$

where $v_{j}=\left(v, e_{j}\right)$.

Our goal in this section is to find sufficient conditions on the operator $A$ and $F$ such that the model problem (2.1) possesses an inertial manifold in $H$. An application to the hyperviscous Navier-Stokes equations (1.1) shall be discussed in the subsequent section. Let us first recall the notion of inertial manifold for a dynamical system (see, e.g., [18, 35]).

Definition 2.1. Let $H$ be a Hilbert space. A subset $\mathcal{M} \subset H$ is called an inertial manifold for a dynamical system in $H$ associated with the semigroup $S(t)$ if the following conditions are satisfied:

(1) $\mathcal{M}$ is invariant with respect to the semigroup $S(t)$, i.e. $S(t) \mathcal{M}=\mathcal{M}$, for all $t \geq 0$;

(2) $\mathcal{M}$ is a finite-dimensional Lipschitz manifold, i.e. there exists a Lipschitz continuous function $\Phi: P_{N} H \rightarrow Q_{N} H$ such that

$$
\mathcal{M}:=\left\{u_{+}+\Phi\left(u_{+}\right), u_{+} \in P_{N} H\right\} .
$$

(3) The exponential tracking property holds, namely, there exist $C, \alpha>0$ such that for every $u_{0} \in H$, there exists $v_{0} \in \mathcal{M}$ such that

$$
\left|S(t) u_{0}-S(t) v_{0}\right| \leq C e^{-\alpha t}\left|u_{0}-v_{0}\right|, \text { for all } t \geq 0 .
$$

2.1. A spectral gap condition. We state the following well-known optimal spectral gap condition which guarantees the existence of inertial manifolds for (2.1) (see [28, 30, 35]).

Theorem 2.2. Let the above assumptions on the operator $A$ hold, and assume $F: H \rightarrow H$ is globally Lipschitz continuous with Lipschitz constant L. Suppose there exists $N \in \mathbb{N}$ such that the following spectral gap condition is satisfied:

$$
\frac{\lambda_{N+1}^{\beta}-\lambda_{N}^{\beta}}{\lambda_{N+1}^{1 / 2}+\lambda_{N}^{1 / 2}}>L
$$

Then (2.1) possesses an $N$-dimensional inertial manifold in the sense of Definition 2.1. 
Remark 2.3. If $\beta>3 / 2$, then the spectral gap condition (2.4) is satisfied if we assume that $\lambda_{j} \rightarrow \infty$ as $j \rightarrow \infty$, and $\lambda_{N+1}-\lambda_{N} \geq 1$ for some large $N$. However, in the critical case $\beta=3 / 2$, the condition (2.4) demands a large spectral gap. Indeed, if $\lambda_{N+1}-\lambda_{N} \geq 2 L$, then (2.4) is valid for $\beta=3 / 2$.

This result with sharp spectral gap condition (2.4) was derived by Miklavcic [28] and Romanov [30] (see also Zelik [35]). The condition (2.4) is optimal in the sense that one can find globally Lipschitz nonlinearity $F$ such that $(2.1)$ does not possess an inertial manifold if (2.4) is violated (cf. [15, 31, 35]).

For the reader's convenience, in Appendix 4.3 we prove a strong cone property provided the optimal spectral gap (2.4) is satisfied, which eventually leads to the existence of inertial manifolds for the model $(2.1)$.

2.2. The spatial averaging scheme. In the above subsection, we have pointed out that, regarding the critical case $\beta=3 / 2$ for the model (2.1), the spectral gap condition (2.4) demands a sufficiently large spectral gap for the operator $A$, which may not be available. This motivates us to consider the model (2.1) in the critical case

$$
u_{t}+A^{3 / 2} u+A^{1 / 2} F(u)=f,\left.\quad u\right|_{t=0}=u_{0},
$$

under the scenario when the eigenvalues of $A$ do not have a sufficiently large gap.

We define the projection operators on the low, high and intermediate Fourier modes, respectively, as follows:

$$
P_{k, N} u:=\sum_{\lambda_{j}<\lambda_{N}-k} u_{j} e_{j}, \quad Q_{k, N} u:=\sum_{\lambda_{j}>\lambda_{N}+k} u_{j} e_{j}, \quad R_{k, N} u:=\sum_{\lambda_{N}-k \leq \lambda_{j} \leq \lambda_{N}+k} u_{j} e_{j},
$$

where $u_{j}=\left(u, e_{j}\right)$, for some $k<\lambda_{N}$.

The following theorem states that if the spectral gap condition for 2.5 (i.e. $\lambda_{N+1}^{3 / 2}-\lambda_{N}^{3 / 2}>$ $\left.L\left(\lambda_{N+1}^{1 / 2}+\lambda_{N}^{1 / 2}\right)\right)$ is not fulfilled, but the spatial averaging condition $[2.6)$ is valid, then a strong cone property can be obtained, which implies the existence of an inertial manifold for $(2.5)$.

Theorem 2.4. Let $u_{1}$ and $u_{2}$ be two solutions of (2.5) in H. Set $v=u_{1}-u_{2}$. Define $V(t)=$ $|q|^{2}-|p|^{2}$ where $p=P_{N} v$ and $q=Q_{N} v$. Assume $F: H \rightarrow H$ is Gateaux differentiable with $\left|F^{\prime}(u)\right|_{\mathcal{L}(H, H)} \leq L$ for all $u \in H$, and for some $L \geq 1$. Suppose there exist $N \in \mathbb{N}$ and $k \in\left[\gamma \log \lambda_{N}, \lambda_{N}\right)$ for some $\gamma \in(0,1]$ such that $\lambda_{N} \geq e^{40 L^{2} / \gamma}$ with $1 \leq \lambda_{N+1}-\lambda_{N} \leq 2 L$, and the spatial averaging condition holds:

$$
\left|R_{k, N} F^{\prime}(u) R_{k, N} v\right| \leq \delta|v|, \text { for all } u \in H,
$$

for some $\delta \leq \frac{1}{50}$. Then the following strong cone property is valid,

$$
\frac{d}{d t} V(t)+\left(\lambda_{N+1}^{3 / 2}+\lambda_{N}^{3 / 2}\right) V(t) \leq-\frac{\lambda_{N}^{1 / 2}}{4}|v(t)|^{2}, \quad \text { for all } t \geq 0 .
$$

Proof. Let $u_{1}$ and $u_{2}$ be two solutions of (2.5) and set $v=u_{1}-u_{2}$, then one has

$$
v_{t}+A^{3 / 2} v+A^{1 / 2}\left[F\left(u_{1}\right)-F\left(u_{2}\right)\right]=0 .
$$


Set $p=P_{N} v$ and $q=Q_{N} v$. Take the scalar product of (2.8) with $p$ and $q$ respectively:

$$
\left\{\begin{array}{l}
\frac{1}{2} \frac{d}{d t}|p|^{2}+\left|A^{3 / 4} p\right|^{2}+\left(F\left(u_{1}\right)-F\left(u_{2}\right), A^{1 / 2} p\right)=0 \\
\frac{1}{2} \frac{d}{d t}|q|^{2}+\left|A^{3 / 4} q\right|^{2}+\left(F\left(u_{1}\right)-F\left(u_{2}\right), A^{1 / 2} q\right)=0 .
\end{array}\right.
$$

By subtracting the two equations in $(2.9)$, we obtain

$$
\frac{d}{d t} V(t)=-2\left(\left|A^{3 / 4} q\right|^{2}-\left|A^{3 / 4} p\right|^{2}\right)+2\left(F\left(u_{1}\right)-F\left(u_{2}\right), A^{1 / 2} p-A^{1 / 2} q\right) .
$$

Due to the fundamental theorem of calculus for the Gateaux derivative, we have

$$
F\left(u_{1}\right)-F\left(u_{2}\right)=\int_{0}^{1} F^{\prime}\left(s u_{1}+(1-s) u_{2}\right) v d s .
$$

Therefore, by setting $\alpha:=\frac{\lambda_{N+1}^{3 / 2}+\lambda_{N}^{3 / 2}}{2}$, and due to 2.10 and 2.11, we have

$$
\begin{aligned}
& \frac{d}{d t} V(t)+2 \alpha V(t)=\left[\alpha V(t)-\left(\left|A^{3 / 4} q\right|^{2}-\left|A^{3 / 4} p\right|^{2}\right)\right]-\left(\left|A^{3 / 4} q\right|^{2}-\alpha|q|^{2}\right) \\
& -\left(\alpha|p|^{2}-\left|A^{3 / 4} p\right|^{2}\right)+2 \int_{0}^{1}\left(F^{\prime}\left(s u_{1}+(1-s) u_{2}\right) v, A^{1 / 2} p-A^{1 / 2} q\right) d s .
\end{aligned}
$$

The idea is to properly bound each of the summands on the right-hand side of (2.12). Since $|v|^{2}=|p|^{2}+|q|^{2}$ and $\left|A^{3 / 4} q\right|^{2} \geq \lambda_{N+1}^{3 / 2}|q|^{2},\left|A^{3 / 4} p\right|^{2} \leq \lambda_{N}^{3 / 2}|p|^{2}$, we have

$$
\begin{aligned}
& \alpha V(t)-\left(\left|A^{3 / 4} q\right|^{2}-\left|A^{3 / 4} p\right|^{2}\right) \\
& \leq \frac{\lambda_{N+1}^{3 / 2}+\lambda_{N}^{3 / 2}}{2}\left(|q|^{2}-|p|^{2}\right)-\lambda_{N+1}^{3 / 2}|q|^{2}+\lambda_{N}^{3 / 2}|p|^{2} \\
& =-\frac{\lambda_{N+1}^{3 / 2}-\lambda_{N}^{3 / 2}}{2}|v|^{2} .
\end{aligned}
$$

For any real numbers $a \geq b \geq 0$, the following elementary inequality holds:

$$
a^{3}-b^{3}=(a-b)\left(a^{2}+a b+b^{2}\right) \geq \frac{1}{2}(a-b)\left(a^{2}+2 a b+b^{2}\right)=\frac{1}{2}\left(a^{2}-b^{2}\right)(a+b) .
$$

Hence,

$$
\frac{\lambda_{N+1}^{3 / 2}-\lambda_{N}^{3 / 2}}{2} \geq \frac{1}{4}\left(\lambda_{N+1}-\lambda_{N}\right)\left(\lambda_{N+1}^{1 / 2}+\lambda_{N}^{1 / 2}\right) \geq \frac{1}{2} \lambda_{N}^{1 / 2},
$$

owing to the assumption $\lambda_{N+1}-\lambda_{N} \geq 1$.

Applying 2.15) to 2.13 yields

$$
\alpha V(t)-\left(\left|A^{3 / 4} q\right|^{2}-\left|A^{3 / 4} p\right|^{2}\right) \leq-\frac{1}{2} \lambda_{N}^{1 / 2}|v|^{2} .
$$


Next notice that $p=P_{k, N} v+R_{k, N} p$. Thus, since $\left|P_{k, N} A^{3 / 4} v\right|^{2} \leq\left(\lambda_{N}-k\right)^{3 / 2}\left|P_{k, N} v\right|^{2}$ and $\left|R_{k, N} A^{3 / 4} p\right|^{2} \leq \lambda_{N}^{3 / 2}\left|R_{k, N} p\right|^{2}$, we have

$$
\begin{aligned}
& \alpha|p|^{2}-\left|A^{3 / 4} p\right|^{2} \\
& =\frac{\lambda_{N+1}^{3 / 2}+\lambda_{N}^{3 / 2}}{2}\left(\left|P_{k, N} v\right|^{2}+\left|R_{k, N} p\right|^{2}\right)-\left(\left|P_{k, N} A^{3 / 4} v\right|^{2}+\left|R_{k, N} A^{3 / 4} p\right|^{2}\right) \\
& \geq\left[\frac{\lambda_{N+1}^{3 / 2}+\lambda_{N}^{3 / 2}}{2}-\left(\lambda_{N}-k\right)^{3 / 2}\right]\left|P_{k, N} v\right|^{2}+\frac{\lambda_{N+1}^{3 / 2}-\lambda_{N}^{3 / 2}}{2}\left|R_{k, N} p\right|^{2} .
\end{aligned}
$$

Since $\lambda_{N+1} \geq \lambda_{N}$ and due the elementary inequality (2.14), one has

$$
\begin{aligned}
\frac{\lambda_{N+1}^{3 / 2}+\lambda_{N}^{3 / 2}}{2}-\left(\lambda_{N}-k\right)^{3 / 2} & \geq \lambda_{N}^{3 / 2}-\left(\lambda_{N}-k\right)^{3 / 2} \\
& \geq \frac{1}{2}\left[\lambda_{N}-\left(\lambda_{N}-k\right)\right]\left[\lambda_{N}^{1 / 2}+\left(\lambda_{N}-k\right)^{1 / 2}\right] \geq \frac{k}{2} \lambda_{N}^{1 / 2} .
\end{aligned}
$$

Thus, applying (2.18) and 2.15) to 2.17) shows that

$$
\alpha|p|^{2}-\left|A^{3 / 4} p\right|^{2} \geq \frac{k}{2} \lambda_{N}^{1 / 2}\left|P_{k, N} v\right|^{2}+\frac{1}{2} \lambda_{N}^{1 / 2}\left|R_{k, N} p\right|^{2} .
$$

Since $q=Q_{k, N} v+R_{k, N} q$, we write $\left|A^{3 / 4} q\right|^{2}-\alpha|q|^{2}$ as follows:

$$
\left|A^{3 / 4} q\right|^{2}-\alpha|q|^{2}=\left(\left|Q_{k, N} A^{3 / 4} v\right|^{2}-\alpha\left|Q_{k, N} v\right|^{2}\right)+\left(\left|R_{k, N} A^{3 / 4} q\right|^{2}-\alpha\left|R_{k, N} q\right|^{2}\right) .
$$

Clearly, $\left|R_{k, N} A^{3 / 4} q\right|^{2} \geq \lambda_{N+1}^{3 / 2}\left|R_{k, N} q\right|^{2}$, then by using 2.15 , we have

$$
\left|R_{k, N} A^{3 / 4} q\right|^{2}-\alpha\left|R_{k, N} q\right|^{2} \geq \frac{\lambda_{N+1}^{3 / 2}-\lambda_{N}^{3 / 2}}{2}\left|R_{k, N} q\right|^{2} \geq \frac{1}{2} \lambda_{N}^{1 / 2}\left|R_{k, N} q\right|^{2}
$$

By the assumption $k \geq \gamma \log \lambda_{N}$ and $\lambda_{N} \geq e^{40 L^{2} / \gamma}$, one has

$$
k \geq 40 L^{2} .
$$

Moreover, note that $\left|Q_{k, N} A^{3 / 4} v\right|^{2} \geq\left(\lambda_{N}+k\right)^{3 / 2}\left|Q_{k, N} v\right|^{2}$. Then using $\alpha=\frac{\lambda_{N+1}^{3 / 2}+\lambda_{N}^{3 / 2}}{2} \leq$ $\lambda_{N+1}^{3 / 2}$, as well as the elementary inequality 2.14 , we obtain

$$
\begin{aligned}
\left|Q_{k, N} A^{3 / 4} v\right|^{2}-\alpha\left|Q_{k, N} v\right|^{2} & \geq\left[\left(\lambda_{N}+k\right)^{3 / 2}-\lambda_{N+1}^{3 / 2}\right]\left|Q_{k, N} v\right|^{2} \\
& \geq \frac{1}{2}\left(\lambda_{N}+k-\lambda_{N+1}\right)\left[\left(\lambda_{N}+k\right)^{1 / 2}+\lambda_{N+1}^{1 / 2}\right]\left|Q_{k, N} v\right|^{2} \\
& \geq(k-2 L) \lambda_{N+1}^{1 / 2}\left|Q_{k, N} v\right|^{2}
\end{aligned}
$$

by the assumption $\lambda_{N+1}-\lambda_{N} \leq 2 L$. Also we have used $\lambda_{N}+k \geq \lambda_{N}+2 L \geq \lambda_{N+1}$, where the first inequality is due to 2.22 as well as $L \geq 1$. 
However, we may find another lower bound for $\left|Q_{k, N} A^{3 / 4} v\right|^{2}-\alpha\left|Q_{k, N} v\right|^{2}$ different from (2.23). Indeed,

$$
\begin{aligned}
& \left|Q_{k, N} A^{3 / 4} v\right|^{2}-\alpha\left|Q_{k, N} v\right|^{2} \\
& \geq \frac{\gamma \log \lambda_{N}}{\lambda_{N}}\left|Q_{k, N} A^{3 / 4} v\right|^{2}+\left[\left(1-\frac{\gamma \log \lambda_{N}}{\lambda_{N}}\right)\left|Q_{k, N} A^{3 / 4} v\right|^{2}-\alpha\left|Q_{k, N} v\right|^{2}\right] \\
& \geq \frac{\gamma \log \lambda_{N}}{\lambda_{N}^{1 / 2}}\left|Q_{k, N} A^{1 / 2} v\right|^{2}+\left[\left(1-\frac{\gamma \log \lambda_{N}}{\lambda_{N}}\right)\left|Q_{k, N} A^{3 / 4} v\right|^{2}-\alpha\left|Q_{k, N} v\right|^{2}\right] .
\end{aligned}
$$

We shall show that the second term on the right-hand side of $(2.24)$ is nonnegative. By the assumptions $\lambda_{N}>k \geq \gamma \log \lambda_{N}$ for some $\gamma \in(0,1]$ and $\lambda_{N+1} \leq \lambda_{N}+2 L$, we evaluate

$$
\begin{aligned}
& \left(1-\frac{\gamma \log \lambda_{N}}{\lambda_{N}}\right)\left|Q_{k, N} A^{3 / 4} v\right|^{2}-\alpha\left|Q_{k, N} v\right|^{2} \\
& \geq\left(1-\frac{\gamma \log \lambda_{N}}{\lambda_{N}}\right)\left(\lambda_{N}+k\right)^{3 / 2}\left|Q_{k, N} v\right|^{2}-\lambda_{N+1}^{3 / 2}\left|Q_{k, N} v\right|^{2} \\
& \geq\left[\left(1-\frac{\gamma \log \lambda_{N}}{\lambda_{N}}\right)\left(\lambda_{N}+\gamma \log \lambda_{N}\right)^{3 / 2}-\left(\lambda_{N}+2 L\right)^{3 / 2}\right]\left|Q_{k, N} v\right|^{2} .
\end{aligned}
$$

Now, we calculate

$$
\begin{aligned}
& \left(1-\frac{\gamma \log \lambda_{N}}{\lambda_{N}}\right)^{2}\left(\lambda_{N}+\gamma \log \lambda_{N}\right)^{3} \\
& =\lambda_{N}^{3}+\lambda_{N}^{2} \gamma \log \lambda_{N}-2 \lambda_{N}\left(\gamma \log \lambda_{N}\right)^{2}-2\left(\gamma \log \lambda_{N}\right)^{3}+\frac{\left(\gamma \log \lambda_{N}\right)^{4}}{\lambda_{N}}+\frac{\left(\gamma \log \lambda_{N}\right)^{5}}{\lambda_{N}^{2}} \\
& \geq \lambda_{N}^{3}+\frac{1}{2} \lambda_{N}^{2} \gamma \log \lambda_{N} \geq \lambda_{N}^{3}+20 L^{2} \lambda_{N}^{2} \geq\left(\lambda_{N}+2 L\right)^{3}
\end{aligned}
$$

since we assume $\lambda_{N} \geq e^{40 L^{2} / \gamma}$, where $L \geq 1$. The inequality 2.26 implies that the righthand side of (2.25) is non-negative, and then due to (2.24), we obtain

$$
\left|Q_{k, N} A^{3 / 4} v\right|^{2}-\alpha\left|Q_{k, N} v\right|^{2} \geq \frac{\gamma \log \lambda_{N}}{\lambda_{N}^{1 / 2}}\left|Q_{k, N} A^{1 / 2} v\right|^{2}
$$

We apply the estimates (2.21), 2.23) and (2.27) to the equality 2.20). Then

$$
\left|A^{3 / 4} q\right|^{2}-\alpha|q|^{2} \geq \frac{\gamma \log \lambda_{N}}{2 \lambda_{N}^{1 / 2}}\left|Q_{k, N} A^{1 / 2} v\right|^{2}+\frac{k-2 L}{2} \lambda_{N+1}^{1 / 2}\left|Q_{k, N} v\right|^{2}+\frac{1}{2} \lambda_{N}^{1 / 2}\left|R_{k, N} q\right|^{2}
$$


In order to deal with the nonlinear term in 2.12 , we evaluate $\left(F^{\prime}(u) v, A^{1 / 2} p-A^{1 / 2} q\right)$ for any $u \in H$ as follows.

$$
\begin{aligned}
( & \left.F^{\prime}(u) v, A^{1 / 2} p-A^{1 / 2} q\right) \\
= & \left(R_{k, N} F^{\prime}(u) v, A^{1 / 2} p-A^{1 / 2} q\right)+\left(P_{k, N} F^{\prime}(u) v, A^{1 / 2} p-A^{1 / 2} q\right) \\
& +\left(Q_{k, N} F^{\prime}(u) v, A^{1 / 2} p-A^{1 / 2} q\right) \\
= & \left(R_{k, N} F^{\prime}(u) R_{k, N} v, A^{1 / 2} p-A^{1 / 2} q\right)+\left(R_{k, N} F^{\prime}(u) P_{k, N} v, A^{1 / 2} p-A^{1 / 2} q\right) \\
& +\left(R_{k, N} F^{\prime}(u) Q_{k, N} v, A^{1 / 2} p-A^{1 / 2} q\right)+\left(P_{k, N} F^{\prime}(u) v, A^{1 / 2} p-A^{1 / 2} q\right) \\
& +\left(Q_{k, N} F^{\prime}(u) v, A^{1 / 2} p-A^{1 / 2} q\right) \\
= & \left(R_{k, N} F^{\prime}(u) R_{k, N} v, A^{1 / 2} p-A^{1 / 2} q\right)+\left(F^{\prime}(u) P_{k, N} v, R_{k, N} A^{1 / 2} p\right) \\
& -\left(F^{\prime}(u) P_{k, N} v, R_{k, N} A^{1 / 2} q\right)+\left(F^{\prime}(u) Q_{k, N} v, R_{k, N} A^{1 / 2} p\right)-\left(F^{\prime}(u) Q_{k, N} v, R_{k, N} A^{1 / 2} q\right) \\
& +\left(F^{\prime}(u) v, P_{k, N} A^{1 / 2} v\right)-\left(F^{\prime}(u) v, Q_{k, N} A^{1 / 2} v\right) .
\end{aligned}
$$

We shall estimate every term on the right-hand side of $2.29 p$. Since $\left|F^{\prime}(u)\right|_{\mathcal{L}(H, H)} \leq L$, we notice that

$$
\begin{aligned}
& \left|\left(F^{\prime}(u) P_{k, N} v, R_{k, N} A^{1 / 2} p\right)\right|+\left|\left(F^{\prime}(u) Q_{k, N} v, R_{k, N} A^{1 / 2} p\right)\right| \\
& \leq L\left(\left|P_{k, N} v\right|+\left|Q_{k, N} v\right|\right)\left|R_{k, N} A^{1 / 2} p\right| \\
& \leq L \lambda_{N}^{1 / 2}\left(\left|P_{k, N} v\right|+\left|Q_{k, N} v\right|\right)\left|R_{k, N} p\right| \\
& \leq L^{2} \lambda_{N}^{1 / 2}\left(\left|P_{k, N} v\right|^{2}+\left|Q_{k, N} v\right|^{2}\right)+\frac{\lambda_{N}^{1 / 2}}{4}\left|R_{k, N} p\right|^{2} .
\end{aligned}
$$

Similarly,

$$
\begin{aligned}
& \left|\left(F^{\prime}(u) P_{k, N} v, R_{k, N} A^{1 / 2} q\right)\right|+\left|\left(F^{\prime}(u) Q_{k, N} v, R_{k, N} A^{1 / 2} q\right)\right| \\
& \leq L\left(\left|P_{k, N} v\right|+\left|Q_{k, N} v\right|\right)\left|R_{k, N} A^{1 / 2} q\right| \\
& \leq L\left(\left|P_{k, N} v\right|+\left|Q_{k, N} v\right|\right)\left(\lambda_{N}+k\right)^{1 / 2}\left|R_{k, N} q\right| \\
& \leq L\left(\left|P_{k, N} v\right|+\left|Q_{k, N} v\right|\right)\left(2 \lambda_{N}\right)^{1 / 2}\left|R_{k, N} q\right| \\
& \leq 4 L^{2} \lambda_{N}^{1 / 2}\left(\left|P_{k, N} v\right|^{2}+\left|Q_{k, N} v\right|^{2}\right)+\frac{\lambda_{N}^{1 / 2}}{8}\left|R_{k, N} q\right|^{2},
\end{aligned}
$$

where we have used the assumption $k<\lambda_{N}$.

Next,

$$
\begin{aligned}
& \left|\left(F^{\prime}(u) v, P_{k, N} A^{1 / 2} v\right)\right| \leq L|v|\left|P_{k, N} A^{1 / 2} v\right| \leq L|v|\left(\lambda_{N}-k\right)^{1 / 2}\left|P_{k, N} v\right| \\
& \leq L|v| \lambda_{N}^{1 / 2}\left|P_{k, N} v\right| \leq 5 L^{2} \lambda_{N}^{1 / 2}\left|P_{k, N} v\right|^{2}+\frac{\lambda_{N}^{1 / 2}}{20}|v|^{2} .
\end{aligned}
$$


Also,

$$
\begin{aligned}
\left|\left(F^{\prime}(u) v, Q_{k, N} A^{1 / 2} v\right)\right| & \leq L\left|v \| Q_{k, N} A^{1 / 2} v\right| \\
& \leq \frac{2 L^{2} \lambda_{N}^{1 / 2}}{\gamma \log \lambda_{N}}|v|^{2}+\frac{\gamma \log \lambda_{N}}{8 \lambda_{N}^{1 / 2}}\left|Q_{k, N} A^{1 / 2} v\right|^{2} .
\end{aligned}
$$

Moreover, by the spatial averaging condition $\left|R_{k, N} F^{\prime}(u) R_{k, N} v\right| \leq \delta|v|$ (see (2.6)), we obtain

$$
\begin{aligned}
& \left|\left(R_{k, N} F^{\prime}(u) R_{k, N} v, A^{1 / 2} p-A^{1 / 2} q\right)\right| \leq \delta|v|\left|A^{1 / 2} p-A^{1 / 2} q\right| \leq \delta|v|\left|A^{1 / 2} p\right|+\delta|v|\left|A^{1 / 2} q\right| \\
& \leq \delta \lambda_{N}^{1 / 2}|v||p|+\delta|v \|| Q_{k, N} A^{1 / 2} v|+\delta| v|| R_{k, N} A^{1 / 2} q \mid \\
& \leq \delta \lambda_{N}^{1 / 2}|v|^{2}+\delta|v|\left|Q_{k, N} A^{1 / 2} v\right|+\delta\left(2 \lambda_{N}\right)^{1 / 2}|v|\left|R_{k, N} q\right| \\
& \leq \delta \lambda_{N}^{1 / 2}|v|^{2}+\frac{2 \delta^{2} \lambda_{N}^{1 / 2}}{\gamma \log \lambda_{N}}|v|^{2}+\frac{\gamma \log \lambda_{N}}{8 \lambda_{N}^{1 / 2}}\left|Q_{k, N} A^{1 / 2} v\right|^{2}+4 \delta^{2} \lambda_{N}^{1 / 2}|v|^{2}+\frac{\lambda_{N}^{1 / 2}}{8}\left|R_{k, N} q\right|^{2} \\
& =\left[\delta+\frac{2 \delta^{2}}{\gamma \log \lambda_{N}}+4 \delta^{2}\right] \lambda_{N}^{1 / 2}|v|^{2}+\frac{\gamma \log \lambda_{N}}{8 \lambda_{N}^{1 / 2}}\left|Q_{k, N} A^{1 / 2} v\right|^{2}+\frac{\lambda_{N}^{1 / 2}}{8}\left|R_{k, N} q\right|^{2} .
\end{aligned}
$$

Substituting (2.30)-(2.34) into (2.29) yields

$$
\begin{aligned}
\mid\left(F^{\prime}(u) v,\right. & \left.A^{1 / 2} p-A^{1 / 2} q\right)\left.\left|\leq\left[\delta+\frac{2\left(\delta^{2}+L^{2}\right)}{\gamma \log \lambda_{N}}+4 \delta^{2}+\frac{1}{20}\right] \lambda_{N}^{1 / 2}\right| v\right|^{2} \\
+ & \frac{\gamma \log \lambda_{N}}{4 \lambda_{N}^{1 / 2}}\left|Q_{k, N} A^{1 / 2} v\right|^{2}+5 L^{2} \lambda_{N}^{1 / 2}\left|Q_{k, N} v\right|^{2}+10 L^{2} \lambda_{N}^{1 / 2}\left|P_{k, N} v\right|^{2} \\
+ & \frac{\lambda_{N}^{1 / 2}}{4}\left|R_{k, N} q\right|^{2}+\frac{\lambda_{N}^{1 / 2}}{4}\left|R_{k, N} p\right|^{2}
\end{aligned}
$$

for any $u \in H$.

Now we apply (2.16), (2.19), 2.28), and (2.35) to the equality (2.12). It follows that

$$
\begin{aligned}
& \frac{d}{d t} V(t)+2 \alpha V(t) \\
& \leq-\left[\frac{1}{2}-2 \delta-\frac{4\left(\delta^{2}+L^{2}\right)}{\gamma \log \lambda_{N}}-8 \delta^{2}-\frac{1}{10}\right] \lambda_{N}^{1 / 2}|v|^{2} \\
& -\left[\frac{k-2 L}{2}-10 L^{2}\right] \lambda_{N}^{1 / 2}\left|Q_{k, N} v\right|^{2}-\left[\frac{k}{2}-20 L^{2}\right] \lambda_{N}^{1 / 2}\left|P_{k, N} v\right|^{2} .
\end{aligned}
$$

By 2.22 we know that $k \geq 40 L^{2}$, where $L \geq 1$, then clearly

$$
\frac{k-2 L}{2}-10 L^{2}>0 \text { and } \frac{k}{2}-20 L^{2} \geq 0 .
$$

Since $\delta \leq 1 / 50$ and $\gamma \log \lambda_{N} \geq 40 L^{2}$ with $L \geq 1$, then

$$
\frac{1}{2}-2 \delta-\frac{4\left(\delta^{2}+L^{2}\right)}{\gamma \log \lambda_{N}}-8 \delta^{2}-\frac{1}{10} \geq \frac{1}{4} .
$$


By 2.36)-(2.37), we conclude that

$$
\frac{d}{d t} V(t)+2 \alpha V(t) \leq-\frac{\lambda_{N}^{1 / 2}}{4}|v|^{2}, \text { for all } t \geq 0
$$

where $\alpha=\frac{\lambda_{N+1}^{3 / 2}+\lambda_{N}^{3 / 2}}{2}$. This completes the proof.

The following result shows that the strong cone property 2.7 implies the cone invariance property and the decay property.

Proposition 2.5. Let $u_{1}$ and $u_{2}$ be two solutions of (2.5) in H. Set $v=u_{1}-u_{2}$. Define $V(t)=|q|^{2}-|p|^{2}$ where $p=P_{N} v$ and $q=Q_{N} v$. Assume $F: H \rightarrow H$ is globally Lipschitz continuous with Lipschitz constant L. Suppose the strong cone property

$$
\frac{d}{d t} V(t)+\alpha V(t) \leq-\mu|v(t)|^{2}, \text { for all } t \geq 0
$$

holds for some constants $\alpha, \mu>0$. Then, the following properties hold.

- Cone invariance property: If $V(0) \leq 0$, then $V(t) \leq 0$ for all $t \geq 0$.

- Decay property: If $V(T)>0$ for some $T>0$, then

$$
|v(t)|^{2} \leq C e^{-\alpha t}|v(0)|^{2}, \quad \text { for all } t \in[0, T],
$$

where $C>0$. (This is also called the squeezing property.)

Remark 2.6. Notice that, the cone invariance property implies that, if $V(T)>0$ for some $T>0$, then $V(t)>0$ for all $t \in[0, T]$.

Proof. The argument adopts the proof of [35, Corollary 2.22]. The cone invariance property follows directly from 2.38). It remains to show the decay property. Indeed, taking the scalar product of 2.8 with $v$ yields

$$
\frac{1}{2} \frac{d}{d t}|v|^{2}+\left|A^{3 / 4} v\right|^{2} \leq\left|\left(F\left(u_{1}\right)-F\left(u_{2}\right), A^{1 / 2} v\right)\right| \leq \frac{L^{2}}{2}|v|^{2}+\frac{\left|A^{1 / 2} v\right|^{2}}{2},
$$

by using the Lipschitz continuity $\left|F\left(u_{1}\right)-F\left(u_{2}\right)\right| \leq L|v|$. Without loss of generality, we assume the eigenvalues $\lambda_{j}$ of $A$ satisfy $\lambda_{j} \geq 1$ for all $j \in \mathbb{N}$, so that $\left|A^{3 / 4} v\right| \geq\left|A^{1 / 2} v\right|$. It follows that

$$
\frac{d}{d t}|v|^{2} \leq L^{2}|v|^{2}
$$

Define $V_{\epsilon}(t)=\epsilon|v(t)|^{2}+V(t)$. Multiply 2.40 by $\epsilon$ and add to (2.38), then

$$
\frac{d}{d t} V_{\epsilon}(t)+\alpha V_{\epsilon}(t) \leq\left[\epsilon\left(\alpha+L^{2}\right)-\mu\right]|v|^{2}=0,
$$

by choosing $\epsilon=\frac{\mu}{\alpha+L^{2}}$. It follows that

$$
V_{\epsilon}(t) \leq e^{-\alpha t} V_{\epsilon}(0), \text { for } t \geq 0 .
$$

If $V(T)>0$ for some $T>0$, then by the cone invariance property, we have $V(t)>0$ for all $t \in[0, T]$. Also, notice that $V(t)=|q|^{2}-|p|^{2} \leq|q|^{2}+|p|^{2}=|v|^{2}$. Therefore, due to (2.41,

$$
\epsilon|v(t)|^{2} \leq \epsilon|v(t)|^{2}+V(t) \leq e^{-\alpha t}\left(\epsilon|v(0)|^{2}+V(0)\right) \leq(\epsilon+1) e^{-\alpha t}|v(0)|^{2},
$$

for all $t \in[0, T]$. Then, $|v(t)|^{2} \leq\left(1+\frac{1}{\epsilon}\right) e^{-\alpha t}|v(0)|^{2}$, for all $t \in[0, T]$. 
It is expected that the cone invariance property along with the decay property should imply the existence of inertial manifolds. This result is stated in Theorem 2.7 below. For the sake of completeness, we refer the reader for a proof of Theorem 2.7 in Appendix 4.2 .

Theorem 2.7. Assume $F: H \rightarrow H$ is globally Lipschitz and $\left|A^{-1 / 4} F(u)\right| \leq C$ for all $u \in H$. Assume that the solutions of (2.5) satisfy the cone invariance property and the decay property stated in Proposition 2.5. Then problem (2.5) possesses an $N$-dimensional inertial manifold $\mathcal{M}$ in the sense of Definition 2.1.

Finally, owing to Proposition 2.5 and Theorem 2.7, we conclude that the spatial averaging condition of Theorem 2.2 guarantees the existence of an inertial manifold for the model problem (2.5). In particular, we have the following corollary.

Corollary 2.8. Suppose the assumptions of Theorem 2.4 are satisfied and $\left|A^{-1 / 4} F(u)\right| \leq C$ for all $u \in H$. Then problem (2.5) possesses an $N$-dimensional inertial manifold $\mathcal{M}$ in the sense of Definition 2.1.

\section{Application to the hyperviscous Navier-Stokes equations}

We aim to employ the theory that has been established on the abstract model (2.1) in Section 2 to prove the existence of an inertial manifold for the hyperviscous Navierstokes equation (1.1) in two or three dimensions. We focus on the scenario when the hyperviscosity $\nu(-\Delta)^{\beta} u$ has the critical power $\beta=3 / 2$, because for the case of $\beta>3 / 2$ the spectral gap condition (2.4) is satisfied. Moreover, we shall concentrate on the case of the three-dimensional spatial domain. Indeed, the two-dimensional setting is much easier to study since the required spectral gap condition (2.4) in Theorem 2.2 is fulfilled by a number theoretical result (Theorem 4.2) due to Richards [29].

Specifically, we consider the incompressible hyperviscous Navier-Stokes equation on the three-dimensional torus:

$$
\left\{\begin{array}{l}
u_{t}+\nu(-\Delta)^{3 / 2} u+(u \cdot \nabla) u+\nabla p=f, \quad(t, x) \in \mathbb{R}_{+} \times \mathbb{T}^{3} \\
\operatorname{div} u=0,\left.\quad u\right|_{t=0}=u_{0} .
\end{array}\right.
$$

Set the phase space $H$ as

$$
H:=\left\{u \in\left(L^{2}\left(\mathbb{T}^{3}\right)\right)^{3}: \int_{\mathbb{T}^{3}} u d x=0, \operatorname{div} u=0\right\} .
$$

We denote by $|\cdot|$ the norm of $H$.

Notice that, if $u \in\left(L^{2}\left(\mathbb{T}^{3}\right)\right)^{3}$, then $u=\sum_{j \in \mathbb{Z}^{3}} \hat{u}_{j} e^{i j \cdot x}$ where $\hat{u}_{j}$ are Fourier coefficients. Thus, $\int_{\mathbb{T}^{3}} u d x=0$ is equivalent to $\hat{u}_{0}=0$. Therefore, if $u \in H$, then

$$
u=\sum_{j \in \mathbb{Z}^{3} \backslash\{0\}} \hat{u}_{j} e^{i j \cdot x} .
$$

We denote by $P_{\sigma}:\left(L^{2}\left(\mathbb{T}^{3}\right)\right)^{3} \rightarrow H$ the Helmholtz-Leray orthogonal projection operator, and by $A=-P_{\sigma} \Delta$ the Stokes operator. Since we work with the periodic space, it is known that

$$
A u=-P_{\sigma} \Delta u=-\Delta u, \quad \text { for all } u \in \mathcal{D}(A) .
$$

The operator $A^{-1}$ is a self-adjoint positive definite compact operator from $H$ to $H$. 
As usual, the Sobolev spaces $H^{s}:=D\left(A^{s / 2}\right), s>0$, with the norm $|\cdot|_{H^{s}}=\left|A^{\frac{s}{2}} \cdot\right|$, are defined as

$$
H^{s}=\left\{u \in H:|u|_{H^{s}}^{2}=\sum_{j \in \mathbb{Z}^{3} \backslash\{0\}}|j|^{2 s}\left|\hat{u}_{j}\right|^{2}<\infty\right\} .
$$

For $s<0$, one defines $H^{s}$ as the completion of $H$ with respect to the corresponding norm $|\cdot|_{H^{s}}$.

For $w_{1}, w_{2} \in H^{1}$, we define the bilinear form

$$
B\left(w_{1}, w_{2}\right)=P_{\sigma}\left(\left(w_{1} \cdot \nabla\right) w_{2}\right) .
$$

Notice that (3.1) can be written in the equivalent form in $H$ :

$$
u_{t}+\nu A^{3 / 2} u+B(u, u)=f,\left.\quad u\right|_{t=0}=u_{0} .
$$

Since the exact value of $\nu$ is not essential to our mathematical analysis, we assume without loss of generality that $\nu=1$. Thus in the following work we consider the equation

$$
u_{t}+A^{3 / 2} u+B(u, u)=f,\left.\quad u\right|_{t=0}=u_{0} .
$$

Given the vectors $u=\left(u_{1}, u_{2}, u_{3}\right), v=\left(v_{1}, v_{2}, v_{3}\right), w=\left(w_{1}, w_{2}, w_{3}\right)$, we denote

$$
b(u, v, w):=(B(u, v), w)=\sum_{m, n=1}^{3} \int_{\mathbb{T}^{3}} u_{m} \partial_{m} v_{n} w_{n} d x
$$

whenever the integrals in (3.5) make sense. It is known that $b(u, v, w)$ has the property

$$
b(u, v, w)=-b(u, w, v),
$$

which implies that $b(u, v, v)=0$.

We state the following global well-posedness result for problem (3.4). The solvability of (3.4) will become clear from the dissipative estimates that we obtain in the subsequent subsection. We choose to omit the proof of Theorem 3.1 since it follows from classical arguments employed for the Navier-Stokes equations (see, e.g., [12, 8]).

Theorem 3.1. Let $f \in H^{-3 / 2}$. Then for every $u_{0} \in H$, problem (3.4) possesses a unique weak solution such that $u \in C([0, T] ; H) \cap L^{2}\left(0, T ; H^{3 / 2}\right)$ for an arbitrary time $T>0$. The weak solution depends continuously on the initial datum in a Lipschitz way.

3.1. Dissipative estimates. This section is devoted to showing that the dynamics of (3.4) has an absorbing ball in $H^{4}$, such that (3.4) possesses a global attractor. Let $S(t): H \rightarrow H$ be the solution semigroup generated by the system (3.4).

Theorem 3.2. Let $f \in H^{1}$. Then $(S, H)$ possesses a compact global attractor $\mathcal{G}$ in the phase space $H$, which is a bounded subset of $H^{4}$.

Proof. We recall that the existence of a finite dimensional global attractor in $H$ for a slightly more general problem than (3.4) is also proven in [3]. Here, for the sake of simplicity we provide the a priori estimates for the existence of a regular absorbing set for the semigroup. The conclusion of the theorem then follows in a standard way (see, e.g. [33]).

In the following estimates, we repeatedly use Hölder's inequality, Young's inequality and the Sobolev imbedding $H^{s} \hookrightarrow L^{\frac{6}{3-2 s}}$ in three dimensions for $s \in(0,3 / 2)$. 
Step 1 ( $L^{2}$-estimate). Take scalar product of 3.4 with $u$. We get

$$
\frac{1}{2} \frac{d}{d t}|u|^{2}+\left|A^{3 / 4} u\right|^{2}=(f, u)=\left(A^{-3 / 4} f, A^{3 / 4} u\right) \leq \frac{1}{2}|f|_{H^{-3 / 2}}^{2}+\frac{1}{2}\left|A^{3 / 4} u\right|^{2} .
$$

It follows that

$$
\frac{d}{d t}|u|^{2}+\left|A^{3 / 4} u\right|^{2} \leq|f|_{H^{-3 / 2}}^{2}
$$

Therefore,

This implies

$$
\frac{d}{d t}|u|^{2}+|u|^{2} \leq|f|_{H^{-3 / 2}}^{2}
$$

Consequently, we have

$$
|u|^{2} \leq|f|_{H^{-3 / 2}}^{2}\left(1-e^{-t}\right)+e^{-t}\left|u_{0}\right|^{2}
$$

$$
\limsup _{t \rightarrow+\infty}|u(t)| \leq|f|_{H^{-3 / 2}} .
$$

Due to (3.9) there exists $t_{0}>0$ such that

$$
|u(t)| \leq 2|f|_{H^{-3 / 2}}, \text { for } t \geq t_{0} .
$$

This shows that all trajectories of $(3.4)$ enter an absorbing ball of radius $2|f|_{H^{-3 / 2}}$ in $H$ for $t \geq t_{0}$.

Step 2 ( $H^{1}$-estimate). Take scalar product of (3.4) with $A u$. We get

$$
\frac{1}{2} \frac{d}{d t}\left|A^{1 / 2} u\right|^{2}+\left|A^{5 / 4} u\right|^{2}+b(u, u, A u)=(f, A u) \leq|f|_{H^{-1 / 2}}^{2}+\frac{1}{4}\left|A^{5 / 4} u\right|^{2} .
$$

We estimate

$$
|b(u, u, A u)| \leq C|u|_{L^{6}}|u|_{H^{1}}|A u|_{L^{3}} \leq C|u|_{H^{1}}^{2}\left|A^{5 / 4} u\right| \leq \frac{1}{4}\left|A^{5 / 4} u\right|^{2}+C|u|_{H^{1}}^{4} .
$$

By (3.11) and (3.12), we see that

$$
\frac{d}{d t}|u|_{H^{1}}^{2}+\left|A^{5 / 4} u\right|^{2} \leq C|u|_{H^{1}}^{4}+2|f|_{H^{-1 / 2}}^{2} .
$$

Notice that, by integrating (3.7) from $t$ to $t+1$, we obtain

$$
\int_{t}^{t+1}|u(\tau)|_{H^{3 / 2}}^{2} d \tau \leq|u(t)|^{2}+|f|_{H^{-3 / 2}}^{2} \leq 5|f|_{H^{-3 / 2}}^{2}
$$

for $t \geq t_{0}$ by using (3.10). By (3.13) and (3.14) along with the uniform Gronwall inequality (see Appendix 4.1, Lemma 4.1), we have

$$
|u(t)|_{H^{1}}^{2} \leq \rho_{1}^{2}:=\exp \left(C|f|_{H^{-3 / 2}}^{2}\right)\left(5|f|_{H^{-3 / 2}}^{2}+2|f|_{H^{-1 / 2}}^{2}\right), \text { for } t \geq t_{0}+1 \text {. }
$$

Therefore, all trajectories of the dynamical system enter an absorbing ball of radius $\rho_{1}$ in $H^{1}$ whenever $t \geq t_{0}+1$.

Step 3 ( $H^{2}$-estimate). Take the scalar product of 3.4 with $A^{2} u$. We deduce

$$
\begin{aligned}
\frac{1}{2} \frac{d}{d t}|A u|^{2}+\left|A^{7 / 4} u\right|^{2} & \leq\left|b\left(u, u, A^{2} u\right)\right|+\left|\left(f, A^{2} u\right)\right| \\
& \leq\left|b\left(u, u, A^{2} u\right)\right|+|f|_{H^{1 / 2}}^{2}+\frac{1}{4}\left|A^{7 / 4} u\right|^{2} .
\end{aligned}
$$


We estimate

$$
\begin{aligned}
\left|b\left(u, u, A^{2} u\right)\right| & \leq C\left(|u|_{H^{1}}\left|A^{1 / 2} u\right|_{L^{6}}\left|A^{3 / 2} u\right|_{L^{3}}+|u|_{L^{6}}\left|A u \| A^{3 / 2} u\right|_{L^{3}}\right) \\
& \leq C|u|_{H^{1}}\left|A u \| A^{7 / 4} u\right| \\
& \leq C \rho_{1}^{2}|A u|^{2}+\frac{1}{4}\left|A^{7 / 4} u\right|^{2},
\end{aligned}
$$

for $t \geq t_{0}+1$, on account of (3.15).

We obtain from (3.16) and (3.17) that, for $t \geq t_{0}+1$,

$$
\frac{d}{d t}|u|_{H^{2}}^{2}+|u|_{H^{7 / 2}}^{2} \leq C \rho_{1}^{2}|u|_{H^{2}}^{2}+2|f|_{H^{1 / 2}}^{2}
$$

In order to find an absorbing ball for $u$ in $H^{2}$, we drop the term $|u|_{H^{7 / 2}}^{2}$ in $(3.18)$ and then integrate the inequality from $s$ to $t+1$ for $t_{0}+1 \leq t \leq s \leq t+1$. One has

$$
|u(t+1)|_{H^{2}}^{2} \leq|u(s)|_{H^{2}}^{2}+C \rho_{1}^{2} \int_{t}^{t+1}|u(\tau)|_{H^{2}}^{2} d \tau+2|f|_{H^{1 / 2}}^{2}
$$

Next, integrating (3.19) with respect to $s$ from $t$ to $t+1$ yields

$$
|u(t+1)|_{H^{2}}^{2} \leq\left(C \rho_{1}^{2}+1\right) \int_{t}^{t+1}|u(\tau)|_{H^{2}}^{2} d \tau+2|f|_{H^{1 / 2}}^{2} .
$$

By integrating (3.13) from $t$ to $t+1$ and using (3.15), we obtain that

$$
\int_{t}^{t+1}|u(\tau)|_{H^{5 / 2}}^{2} d \tau \leq \rho_{1}^{2}+C \rho_{1}^{4}+2|f|_{H^{-1 / 2}}^{2}
$$

for $t \geq t_{0}+1$.

By virtue of (3.20) and (3.21), we see that

$$
|u(t)|_{H^{2}}^{2} \leq \rho_{2}^{2}:=C\left(\rho_{1}^{6}+\rho_{1}^{2}|f|_{H^{-1 / 2}}^{2}\right)+2|f|_{H^{1 / 2}}^{2}, \text { for } t \geq t_{0}+2 .
$$

This implies that any solution of (3.4) enters an absorbing ball of radius $\rho_{2}$ in $H^{2}$ after a sufficiently large time $t \geq t_{0}+2$.

Step 4 ( $H^{4}$-estimate). Recall our assumption $f \in H^{1}$. Then, from equation (3.4), we have

$$
A^{1 / 2} u_{t}+A^{2} u+A^{1 / 2} B(u, u)=A^{1 / 2} f \text {, in } H .
$$

Since $\left|A^{1 / 2} B(u, u)\right| \leq C|u|_{H^{2}}^{2}$, we obtain from (3.23) and (3.22) that

$$
|u|_{H^{4}}=\left|A^{2} u\right| \leq\left|u_{t}\right|_{H^{1}}+C|u|_{H^{2}}^{2}+|f|_{H^{1}} \leq\left|u_{t}\right|_{H^{1}}+C \rho_{2}^{2}+|f|_{H^{1}}, \text { for } t \geq t_{0}+2 \text {. }
$$

It remains to show that $\left|u_{t}\right|_{H^{1}}$ has a uniform bound for large time. To this end, we take the scalar product of (3.4) with $A u_{t}$ :

$$
\begin{aligned}
& \left|u_{t}\right|_{H^{1}}^{2}+\frac{1}{2} \frac{d}{d t}|u|_{H^{5 / 2}}^{2}=-b\left(u, u, A u_{t}\right)+\left(f, A u_{t}\right) \\
& \leq C|u|_{H^{2}}^{2}\left|u_{t}\right|_{H^{1}}+|f|_{H^{1}}\left|u_{t}\right|_{H^{1}} \leq \frac{1}{2}\left|u_{t}\right|^{2}+C \rho_{2}^{4}+|f|_{H^{1}}^{2}, \text { for } t \geq t_{0}+2 .
\end{aligned}
$$

Hence,

$$
\left|u_{t}\right|_{H^{1}}^{2}+\frac{d}{d t}|u|_{H^{5 / 2}}^{2} \leq C \rho_{2}^{4}+2|f|_{H^{1}}^{2}, \text { for } t \geq t_{0}+2
$$


Integrate (3.25) over $[s, t+1]$ for $t_{0}+2 \leq t \leq s \leq t+1$ :

$$
|u(t+1)|_{H^{5 / 2}}^{2} \leq|u(s)|_{H^{5 / 2}}^{2}+C \rho_{2}^{4}+2|f|_{H^{1}}^{2} .
$$

Integrating $(3.26)$ over $[t, t+1]$ with respect to $s$ yields

$$
\begin{aligned}
& |u(t+1)|_{H^{5 / 2}}^{2} \leq \int_{t}^{t+1}|u(s)|_{H^{5 / 2}}^{2} d s+C \rho_{2}^{4}+2|f|_{H^{1}}^{2} \\
& \leq \rho_{3}^{2}:=\rho_{1}^{2}+C \rho_{1}^{4}+2|f|_{H^{-1 / 2}}^{2}+C \rho_{2}^{4}+2|f|_{H^{1}}^{2}, \text { for } t \geq t_{0}+2,
\end{aligned}
$$

due to estimate (3.21). Now integrating (3.25) over $[t, t+1]$ for $t \geq t_{0}+3$ and using (3.27), we obtain

$$
\int_{t}^{t+1}\left|u_{t}\right|_{H^{1}}^{2} d \tau \leq \rho_{3}^{2}+C \rho_{2}^{4}+2|f|_{H^{1}}^{2}, \text { for } t \geq t_{0}+3
$$

Next we differentiate (3.4) in time,

$$
u_{t t}+A^{3 / 2} u_{t}+B\left(u_{t}, u\right)+B\left(u, u_{t}\right)=0 .
$$

By taking the scalar product of $(3.29)$ with $A u_{t}$, we have

$$
\frac{1}{2} \frac{d}{d t}\left|u_{t}\right|_{H^{1}}^{2}+\left|u_{t}\right|_{H^{5 / 2}}^{2}=-b\left(u_{t}, u, A u_{t}\right)-b\left(u, u_{t}, A u_{t}\right) \leq C\left|u_{t}\right|_{H^{5 / 2}}\left|u_{t}\right|_{H^{1}}|u|_{H^{1}} .
$$

It follows that

$$
\frac{d}{d t}\left|u_{t}\right|_{H^{1}}^{2}+\left|u_{t}\right|_{H^{5 / 2}}^{2} \leq C\left|u_{t}\right|_{H^{1}}^{2}|u|_{H^{1}}^{2} \leq C \rho_{1}^{2}\left|u_{t}\right|_{H^{1}}^{2}, \text { for } t \geq t_{0}+1 .
$$

Owing to 3.30 and 3.28 , we deduce

$$
\left|u_{t}\right|_{H^{1}}^{2} \leq\left(C \rho_{1}^{2}+1\right)\left(\rho_{3}^{2}+C \rho_{2}^{4}+2|f|_{H^{1}}^{2}\right), \text { for } t \geq t_{0}+4 .
$$

Finally, by virtue of (3.24) and (3.31), we conclude

$$
|u|_{H^{4}} \leq \rho:=C\left(\rho_{1}+1\right)\left(\rho_{3}+\rho_{2}^{2}+|f|_{H^{1}}\right), \text { for } t \geq t_{0}+4 \text {. }
$$

That is, all trajectories of (3.4) enter an absorbing ball in $H^{4}$ with the radius $\rho$ when $t \geq t_{0}+4$ if $f \in H^{1}$. The proof is complete.

Remark 3.3. The existence of an absorbing ball in $H^{4}$ is crucial for the construction of an inertial manifold for (3.4) as we modify the nonlinearity outside the absorbing ball and show that the modified nonlinearity satisfies the spatial averaging condition in the subsequent sections. On the other hand, the global well-posedness of weak solutions and the existence of a global attractor for equation (1.1) for any $\beta \geq 5 / 4$ in three dimensions can be shown by using the standard energy method. For the sake of completion, we briefly discuss in Appendix 4.4 the case $\beta \geq 5 / 4$ for 1.1 in $3 \mathrm{D}$. 
3.2. Modification of the nonlinearity outside the absorbing ball. Since we are concerned with the large-time behavior of the hyperviscous Navier-Stokes equations, we may freely modify the nonlinearity outside the absorbing ball in $H^{4}$. In this section we adopt the method by Kostianko [24] to truncate the nonlinearity of our system (3.4). To this end, let us introduce a smooth cut-off function $\theta \in C_{0}^{\infty}(\mathbb{C})$ satisfying

$$
\theta(\xi)=\xi \text { for }|\xi| \leq 1 \text { and }|\theta(\xi)| \leq 2 \text { for all } \xi \in \mathbb{C}
$$

Also, we define the corresponding vector-valued cut-off function as

$$
\vec{\theta}:=\left(\theta\left(\xi_{1}\right), \theta\left(\xi_{2}\right), \theta\left(\xi_{3}\right)\right) \in \mathbb{C}^{3}, \text { for } \xi=\left(\xi_{1}, \xi_{2}, \xi_{3}\right) \in \mathbb{C}^{3} .
$$

Recall that $H$ denotes the $L^{2}$ divergence-free vector field with zero mean value. The Helmholtz-Leray orthogonal projector $P_{\sigma}:\left(L^{2}\left(\mathbb{T}^{3}\right)\right)^{3} \rightarrow H$ is defined as

$$
P_{\sigma} u=\sum_{j \in \mathbb{Z}^{3} \backslash\{0\}} P_{j} \hat{u}_{j} e^{i j \cdot x}
$$

where the $3 \times 3$ matrices $P_{j}$ are given by

$$
P_{j}:=\frac{1}{|j|^{2}}\left(\begin{array}{ccc}
j_{2}^{2}+j_{3}^{2} & -j_{1} j_{2} & -j_{1} j_{3} \\
-j_{1} j_{2} & j_{1}^{2}+j_{3}^{2} & -j_{2} j_{3} \\
-j_{1} j_{3} & -j_{2} j_{3} & j_{1}^{2}+j_{2}^{2}
\end{array}\right) .
$$

$P_{j}$ are orthonormal projectors in $\mathbb{C}^{3}$.

Following the idea in [24], we introduce the operator $U: H \rightarrow H$ defined as

$$
U(u):=\sum_{j \in \mathbb{Z}^{3} \backslash\{0\}} \frac{\rho}{|j|^{4}} P_{j} \vec{\theta}\left(\frac{|j|^{4} \hat{u}_{j}}{\rho}\right) e^{i j \cdot x},
$$

where $\rho$ is the radius of the absorbing ball in $H^{4}$ established in 3.32 for the system 3.4 . We remark that, in two dimensions, the operator $U$ can be defined in a similar way.

In order to modify the nonlinearity $B(u, u)$ in 3.4 outside the absorbing ball in $H^{4}$, we shall replace $B(u, u)$ by $B(U(u), U(u))$, and then prove the existence of an inertial manifold in $H$ for the so-called "prepared" equation for (3.4), namely

$$
u_{t}+A^{3 / 2} u+B(U(u), U(u))=f,
$$

for $f \in H^{1}$. Indeed we shall see that the original equation (3.4) and the "prepared" equation (3.34) have the same large time behavior in the absorbing ball in $H^{4}$.

For the sake of preparation, we prove a few useful properties of the operator $U$.

Lemma 3.4. The function $U: H \rightarrow H$ has the following properties.

(1) $U(u)=u$, for all $u \in \mathcal{D}\left(A^{2}\right)$ with $|u|_{H^{4}} \leq \rho$.

(2) $U$ is a regularization operator: for any $\delta>0, U: H \rightarrow H^{\frac{5}{2}-\delta}$ and there exists a constant $C_{\delta}$ such that

$$
|U(u)|_{H^{\frac{5}{2}-\delta}} \leq C_{\delta}, \text { for all } u \in H .
$$

Moreover, the map $U: H \rightarrow H^{\frac{5}{2}-\delta}$ is continuous. 
(3) $U$ is Gateaux differentiable from $H$ to $H$, and its derivative $U^{\prime}$ has the expression

$$
U^{\prime}(u) v=\sum_{j \in \mathbb{Z}^{3} \backslash\{0\}} P_{j} \vec{\theta}^{\prime}\left(\frac{|j|^{4} \hat{u}_{j}}{\rho}\right) \hat{v}_{j} e^{i j \cdot x}, \text { for } u, v \in H .
$$

Moreover, there exists $L_{1}>0$ such that

$$
\left|U^{\prime}(u)\right|_{\mathcal{L}(H, H)} \leq L_{1}, \quad \text { for all } u \in H
$$

Furthermore, for each $v \in H$, the map $u \mapsto U^{\prime}(u) v$ is continuous from $H$ to $H$.

Proof. The proof is similar to the arguments in [24].

(1) Let $u \in \mathcal{D}\left(A^{2}\right)$ such that

$$
|u|_{H^{4}}^{2}=\sum_{j \in \mathbb{Z}^{3} \backslash\{0\}}|j|^{8}\left|\hat{u}_{j}\right|^{2} \leq \rho^{2}
$$

then obviously,

$$
\frac{|j|^{4}\left|\hat{u}_{j}\right|}{\rho} \leq 1, \text { for all } j \in \mathbb{Z}^{3} \backslash\{0\}
$$

Since $\theta(\xi)=\xi$ for $|\xi| \leq 1$, one has $\vec{\theta}\left(\frac{|j|^{4} \hat{u}_{j}}{\rho}\right)=\frac{|j|^{4} \hat{u}_{j}}{\rho}$ for all $j \in \mathbb{Z}^{3} \backslash\{0\}$. Hence by the definition (3.33), we see that $U(u)=u$ provided $|u|_{H^{4}} \leq \rho$.

(2) Let $u \in H$. Since $|\theta(\xi)| \leq 2$ for all $\xi \in \mathbb{C}$, then by (3.33), we calculate

$$
|U(u)|_{H^{\frac{5}{2}-\delta}}^{2} \leq C \sum_{j \in \mathbb{Z}^{3} \backslash\{0\}}|j|^{5-2 \delta} \frac{\rho^{2}}{|j|^{8}} \leq C \rho^{2} \sum_{j \in \mathbb{Z}^{3} \backslash\{0\}} \frac{1}{|j|^{3+2 \delta}} \leq C_{\delta} .
$$

Next we show that $U: H \rightarrow H^{\frac{5}{2}-\delta}$ is continuous. Indeed, given $\epsilon>0$, let $u, v \in H$ such that $|u-v| \leq \sigma$. We estimate

$$
\begin{aligned}
& |U(u)-U(v)|_{H^{\frac{5}{2}-\delta}}^{2} \\
& =\sum_{j \in \mathbb{Z}^{3} \backslash\{0\}} \frac{\rho^{2}}{|j|^{3+2 \delta}}\left|\vec{\theta}\left(\frac{|j|^{4} \hat{u}_{j}}{\rho}\right)-\vec{\theta}\left(\frac{|j|^{4} \hat{v}_{j}}{\rho}\right)\right|^{2} \\
& =\sum_{1 \leq|j| \leq N} \frac{\rho^{2}}{|j|^{3+2 \delta}}\left|\vec{\theta}\left(\frac{|j|^{4} \hat{u}_{j}}{\rho}\right)-\vec{\theta}\left(\frac{|j|^{4} \hat{v}_{j}}{\rho}\right)\right|^{2}+C \sum_{|j|>N} \frac{\rho^{2}}{|j|^{3+2 \delta}},
\end{aligned}
$$

where we have used the fact that $\vec{\theta}$ is uniformly bounded in $\mathbb{C}^{3}$. Now we choose $N$ sufficiently large such that $C \sum_{|j|>N} \frac{\rho^{2}}{|j|^{3+2 \delta}} \leq \frac{\epsilon}{2}$. Next, since $|u-v| \leq \sigma$, one has $\left|\hat{u}_{j}-\hat{v}_{j}\right| \leq \sigma$ for every $j \in \mathbb{Z}^{3} \backslash\{0\}$, then by the uniform continuity of $\vec{\theta}$, we may select $\sigma>0$ small enough such that the 1 st summation on the right-hand side of $(3.38)$ is bounded by $\frac{\epsilon}{2}$. 
(3) Let $u, v \in H$. We calculate

$$
\begin{aligned}
& \frac{U(u+\varepsilon v)-U(u)}{\varepsilon} \\
& =\frac{1}{\varepsilon} \sum_{j \in \mathbb{Z}^{3} \backslash\{0\}} \frac{\rho}{|j|^{4}} P_{j}\left[\vec{\theta}\left(\frac{|j|^{4}\left(\hat{u}_{j}+\varepsilon \hat{v}_{j}\right)}{\rho}\right)-\vec{\theta}\left(\frac{|j|^{4} \hat{u}_{j}}{\rho}\right)\right] e^{i j \cdot x} \\
& =\sum_{j \in \mathbb{Z}^{3} \backslash\{0\}} P_{j}\left[\hat{v}_{j} \int_{0}^{1} \vec{\theta}^{\prime}\left(\frac{|j|^{4}\left(\hat{u}_{j}+\tau \varepsilon \hat{v}_{j}\right)}{\rho}\right) d \tau\right] e^{i j \cdot x}
\end{aligned}
$$

where we have used the fundamental theorem of calculus.

If $U^{\prime}(u) v$ is given by 3.36 , we aim to show that the $L^{2}$-norm of the difference of 3.36 and $(3.39)$ converges to zero if $\varepsilon \rightarrow 0$. Indeed,

$$
\begin{aligned}
& \left|\frac{U(u+\varepsilon v)-U(u)}{\varepsilon}-U^{\prime}(u) v\right|_{H}^{2} \\
& =\left|\sum_{j \in \mathbb{Z}^{3} \backslash\{0\}} P_{j}\left[\hat{v}_{j} \int_{0}^{1}\left(\vec{\theta}^{\prime}\left(\frac{|j|^{4}\left(\hat{u}_{j}+\tau \varepsilon \hat{v}_{j}\right)}{\rho}\right)-\vec{\theta}^{\prime}\left(\frac{|j|^{4} \hat{u}_{j}}{\rho}\right)\right) d \tau\right] e^{i j \cdot x}\right|_{H}^{2} \\
& \leq \sum_{j \in \mathbb{Z}^{3} \backslash\{0\}}\left|\hat{v}_{j}\right|^{2} \int_{0}^{1}\left|\vec{\theta}^{\prime}\left(\frac{|j|^{4}\left(\hat{u}_{j}+\tau \varepsilon \hat{v}_{j}\right)}{\rho}\right)-\vec{\theta}^{\prime}\left(\frac{|j|^{4} \hat{u}_{j}}{\rho}\right)\right|^{2} d \tau \\
& \leq \sum_{1 \leq|j| \leq N}\left|\hat{v}_{j}\right|^{2} \int_{0}^{1}\left|\vec{\theta}^{\prime}\left(\frac{|j|^{4}\left(\hat{u}_{j}+\tau \varepsilon \hat{v}_{j}\right)}{\rho}\right)-\vec{\theta}^{\prime}\left(\frac{|j|^{4} \hat{u}_{j}}{\rho}\right)\right|^{2} d \tau+C \sum_{|j|>N}\left|v_{j}\right|^{2},
\end{aligned}
$$

where we have used $\vec{\theta}$ is uniformly bounded in $\mathbb{C}^{3}$. Let $\delta>0$. Since $v \in H$, we choose $N$ sufficiently large such that the $C \sum_{|j|>N}\left|v_{j}\right|^{2} \leq \frac{\delta}{2}$. Also, since $\vec{\theta}^{\prime}$ is uniformly continuous, we can select $\epsilon$ small enough such that the 1 st summation is less than $\frac{\delta}{2}$. This implies

$$
\left|\frac{U(u+\varepsilon v)-U(u)}{\varepsilon}-U^{\prime}(u) v\right|_{H}^{2} \leq \delta,
$$

for sufficiently small $\epsilon$, provided $U^{\prime}(u) v$ is given by (3.36). Therefore, we conclude that (3.36) is the correct expression for $U^{\prime}(u) v$.

Next, by 3.36 and the uniform boundedness of $\vec{\theta}^{\prime}$, there exists $L_{1}>0$ such that $\left|U^{\prime}(u) v\right| \leq L_{1}|v|$, for all $u, v \in H$, i.e., $\left|U^{\prime}(u)\right|_{\mathcal{L}(H, H)} \leq L_{1}$ for all $u \in H$. 
Finally, let $v \in H$, we show that the map $u \mapsto U^{\prime}(u) v$ is continuous from $H$ to $H$. Indeed, given $\epsilon>0$, we let $u, w \in H$ such that $|u-w| \leq \sigma$. Due to $(3.36)$, we have

$$
\begin{aligned}
& \left|\left(U^{\prime}(u)-U^{\prime}(w)\right) v\right|^{2} \\
& =\sum_{j \in \mathbb{Z}^{3} \backslash\{0\}}\left|\vec{\theta}^{\prime}\left(\frac{|j|^{4} \hat{u}_{j}}{\rho}\right)-\vec{\theta}^{\prime}\left(\frac{|j|^{4} \hat{w}_{j}}{\rho}\right)\right|^{2}\left|\hat{v}_{j}\right|^{2} \\
& \leq \sum_{1 \leq|j| \leq N}\left|\vec{\theta}^{\prime}\left(\frac{|j|^{4} \hat{u}_{j}}{\rho}\right)-\vec{\theta}^{\prime}\left(\frac{|j|^{4} \hat{w}_{j}}{\rho}\right)\right|^{2}\left|\hat{v}_{j}\right|^{2}+C \sum_{|j|>N}\left|\hat{v}_{j}\right|^{2},
\end{aligned}
$$

where we have used $\vec{\theta}$ is uniformly bounded in $\mathbb{C}^{3}$. Since $v \in H$, we may choose $N$ sufficiently large such that $C \sum_{|j|>N}\left|\hat{v}_{j}\right|^{2} \leq \frac{\epsilon}{2}$. Also, since $|u-w| \leq \sigma$, one has $\left|\hat{u}_{j}-\hat{w}_{j}\right| \leq \sigma$ for any $j \in \mathbb{Z}^{3} \backslash\{0\}$, and then by the uniform continuity of $\vec{\theta}^{\prime}$, we may select $\sigma>0$ small enough such that $\sum_{1 \leq|j| \leq N}\left|\overrightarrow{\theta^{\prime}}\left(\frac{|j|^{4} \hat{u}_{j}}{\rho}\right)-\vec{\theta}^{\prime}\left(\frac{|j|^{4} \hat{w}_{j}}{\rho}\right)\right|^{2}\left|\hat{v}_{j}\right|^{2} \leq \frac{\epsilon}{2}$. Therefore, we have $\left|\left(U^{\prime}(u)-U^{\prime}(w)\right) v\right|^{2} \leq$ $\epsilon$ if $|u-w| \leq \sigma$.

3.3. Verification of the spatial averaging condition. This subsection is devoted to verifying the spatial averaging condition for the "prepared" equation (3.34). In fact, in order to employ our theory established on the abstract model (2.5), we write the hyperviscous Navier-Stokes equations (3.4) in the form of (2.5):

$$
u_{t}+A^{3 / 2} u+A^{1 / 2} F(u)=f, \text { where } F(u):=A^{-1 / 2} B(u, u) .
$$

Notice that, for the operator $A=-\Delta$, the eigenvector $e^{i j \cdot x}$ corresponds to the eigenvalue $|j|^{2}$. We modify $F(u)$ outside the absorbing ball in $H^{4}$ by replacing $u$ by $U(u)$. Namely, we define $\mathscr{F}: H \rightarrow H$ by

$$
\mathscr{F}(u):=F(U(u))=A^{-1 / 2} B(U(u), U(u)) .
$$

By Lemma 3.4, for any $u \in \mathcal{D}\left(A^{2}\right)$ such that $|u|_{H^{4}} \leq \rho$, one has $U(u)=u$, and thus $\mathscr{F}(u)=F(U(u))=F(u)$ if $|u|_{H^{4}} \leq \rho$. Hence, after the trajectory $u(t)$ enters the absorbing ball in $H^{4}$ with radius $\rho$, the equation $(3.42)$ is equivalent to

$$
u_{t}+A^{3 / 2} u+A^{1 / 2} \mathscr{F}(u)=f, \text { where } \mathscr{F}(u)=A^{-1 / 2} B(U(u), U(u)),
$$

for $f \in H^{1}$. Recall that 3.44$)$ is named the "prepared" equation for 3.42 , since equations (3.42) and 3.44 have the same large time behavior in the absorbing ball in $H^{4}$.

Before showing that $\mathscr{F}$ is Gateaux differentiable from $H$ to $H$ (see Proposition 3.5 below), we formally compute the Gateaux derivative of $\mathscr{F}: H \rightarrow H$ at the point $u \in H$ in the direction of $v \in H$. In fact, for any $w \in H$,

$$
\begin{aligned}
\left(\mathscr{F}^{\prime}(u) v, w\right) & =\lim _{\varepsilon \rightarrow 0}\left(\frac{\mathscr{F}(u+\varepsilon v)-\mathscr{F}(u)}{\varepsilon}, w\right) \\
& =\lim _{\varepsilon \rightarrow 0}\left(\frac{B(U(u+\varepsilon v), U(u+\varepsilon v))-B(U(u), U(u))}{\varepsilon}, A^{-1 / 2} w\right) \\
& =\left(B\left(U(u), U^{\prime}(u) v\right), A^{-1 / 2} w\right)+\left(B\left(U^{\prime}(u) v, U(u)\right), A^{-1 / 2} w\right) \\
& =-b\left(U(u), A^{-1 / 2} w, U^{\prime}(u) v\right)-b\left(U^{\prime}(u) v, A^{-1 / 2} w, U(u)\right),
\end{aligned}
$$


where we have used (3.5) and (3.6).

Proposition 3.5. Let $\mathscr{F}$ be the operator defined by (3.43). Then $\mathscr{F}$ is uniformly bounded from $H$ to $H^{2}$, i.e, there exists $C>0$ with $|\mathscr{F}(u)|_{H^{2}} \leq C$ for all $u \in H$. Moreover, $\mathscr{F}$ is Gateaux differentiable from $H$ to $H$, and its derivative $\mathscr{F}^{\prime}$ has the expression:

$$
\left(\mathscr{F}^{\prime}(u) v, w\right)=-b\left(U(u), A^{-1 / 2} w, U^{\prime}(u) v\right)-b\left(U^{\prime}(u) v, A^{-1 / 2} w, U(u)\right),
$$

for $u, v, w \in H$. In addition, there exists $L>0$ such that

$$
\left|\mathscr{F}^{\prime}(u)\right|_{\mathcal{L}(H, H)} \leq L, \text { for all } u \in H .
$$

Proof. To show that $\mathscr{F}$ is uniformly bounded from $H$ to $H^{2}$, we take an arbitrary $w \in H$ and estimate

$$
\begin{aligned}
(A \mathscr{F}(u), w) & =\left(A^{1 / 2} B(U(u), U(u)), w\right) \\
& \leq C|w|\left(\left|A^{1 / 2} U(u)\right|_{L^{4}}^{2}+|U(u)|_{L^{\infty}}|A U(u)|\right) \\
& \leq C|w||U(u)|_{H^{2}}^{2} .
\end{aligned}
$$

Thus $|A \mathscr{F}(u)| \leq|U(u)|_{H^{2}}^{2} \leq C$, for all $u \in H$, by virtue of 3.35 .

Next, we show that $\mathscr{F}$ is Gateaux differentiable from $H$ to $H$. Indeed, for any $u, v$, $w \in H$, we calculate

$$
\begin{aligned}
\left(\frac{\mathscr{F}(u+\varepsilon v)-\mathscr{F}(u)}{\varepsilon}, w\right)= & \left(\frac{B(U(u+\varepsilon v), U(u+\varepsilon v))-B(U(u), U(u))}{\varepsilon}, A^{-1 / 2} w\right) \\
= & -b\left(U(u+\varepsilon v), A^{-1 / 2} w, \frac{U(u+\varepsilon v)-U(u)}{\varepsilon}\right) \\
& -b\left(\frac{U(u+\varepsilon v)-U(u)}{\varepsilon}, A^{-1 / 2} w, U(u)\right),
\end{aligned}
$$

where we have used (3.5) and (3.6).

In order to show that $b\left(U(u+\varepsilon v), A^{-1 / 2} w, \frac{U(u+\varepsilon v)-U(u)}{\varepsilon}\right) \rightarrow b\left(U(u), A^{-1 / 2} w, U^{\prime}(u) v\right)$ as $\varepsilon \rightarrow 0$, we evaluate the difference:

$$
\begin{aligned}
& \left|b\left(U(u+\varepsilon v), A^{-1 / 2} w, \frac{U(u+\varepsilon v)-U(u)}{\varepsilon}\right)-b\left(U(u), A^{-1 / 2} w, U^{\prime}(u) v\right)\right| \\
& \leq\left|b\left(U(u+\varepsilon v)-U(u), A^{-1 / 2} w, \frac{U(u+\varepsilon v)-U(u)}{\varepsilon}\right)\right| \\
& \quad+\left|b\left(U(u), A^{-1 / 2} w, \frac{U(u+\varepsilon v)-U(u)}{\varepsilon}-U^{\prime}(u) v\right)\right| .
\end{aligned}
$$

We shall show that the right-hand side of (3.48) converges to zero. Since $U$ is Gateaux differentiable and the map $u \mapsto U^{\prime}(u) v$ is continuous from $H$ to $H$ due to Lemma 3.4, by the fundamental theorem of calculus for the Gateaux derivative, we have

$$
U(u+\varepsilon v)-U(u)=\int_{0}^{1} U^{\prime}(u+\tau \varepsilon v) \varepsilon v d \tau .
$$


As a result,

$$
\begin{aligned}
& \left|b\left(U(u+\varepsilon v)-U(u), A^{-1 / 2} w, \frac{U(u+\varepsilon v)-U(u)}{\varepsilon}\right)\right| \\
& \leq\left|b\left(U(u+\varepsilon v)-U(u), A^{-1 / 2} w, \int_{0}^{1} U^{\prime}(u+\tau \varepsilon v) v d \tau\right)\right| \\
& \leq C|U(u+\varepsilon v)-U(u)|_{L^{\infty}}|w|\left|\int_{0}^{1} U^{\prime}(u+\tau \varepsilon v) v d \tau\right|_{H} \\
& \leq C|U(u+\varepsilon v)-U(u)|_{L^{\infty}}|w| \int_{0}^{1}\left|U^{\prime}(u+\tau \varepsilon v) v\right|_{H} d \tau \\
& \leq C L_{1}|U(u+\varepsilon v)-U(u)|_{H^{2}}|w||v|
\end{aligned}
$$

where we have applied (3.37). Also, by Lemma 3.4, the map $U: H \rightarrow H^{2}$ is continuous, thus $|U(u+\varepsilon v)-U(u)|_{H^{2}} \rightarrow 0$ as $\varepsilon \rightarrow 0$. It follows that the right-hand side of (3.50) converges to zero as $\varepsilon \rightarrow 0$.

Next, we deal with the second term on the right-hand side of (3.48). Due to (3.49), we see that

$$
\begin{aligned}
& \left|b\left(U(u), A^{-1 / 2} w, \frac{U(u+\varepsilon v)-U(u)}{\varepsilon}-U^{\prime}(u) v\right)\right| \\
& \leq\left|b\left(U(u), A^{-1 / 2} w, \int_{0}^{1}\left(U^{\prime}(u+\tau \varepsilon v)-U^{\prime}(u)\right) v d \tau\right)\right| \\
& \leq C|U(u)|_{L^{\infty}}|w|\left|\int_{0}^{1}\left(U^{\prime}(u+\tau \varepsilon v)-U^{\prime}(u)\right) v d \tau\right|_{H} \\
& \leq C|U(u)|_{H^{2}}|w| \int_{0}^{1}\left|\left(U^{\prime}(u+\tau \varepsilon v)-U^{\prime}(u)\right) v\right|_{H} d \tau .
\end{aligned}
$$

According to Lemma 3.4 the map $u \mapsto U^{\prime}(u) v$ is continuous from $H$ to $H$. Therefore, $\sup _{\tau \in[0,1]}\left|\left(U^{\prime}(u+\tau \varepsilon v)-U^{\prime}(u)\right) v\right|_{H} \rightarrow 0$ as $\varepsilon \rightarrow 0$. It follows that the right-hand side of (3.51) converges to zero as $\varepsilon \rightarrow 0$.

We have shown that both terms on the right-hand side of 3.48 converge to zero as $\varepsilon \rightarrow 0$. Hence

$$
\lim _{\varepsilon \rightarrow 0} b\left(U(u+\varepsilon v), A^{-1 / 2} w, \frac{U(u+\varepsilon v)-U(u)}{\varepsilon}\right)=b\left(U(u), A^{-1 / 2} w, U^{\prime}(u) v\right) .
$$

Analogously, we may show that

$$
\lim _{\varepsilon \rightarrow 0} b\left(\frac{U(u+\varepsilon v)-U(u)}{\varepsilon}, A^{-1 / 2} w, U(u)\right)=b\left(U^{\prime}(u) v, A^{-1 / 2} w, U(u)\right) .
$$

By (3.47), 3.52 and (3.53), we conclude that

$$
\lim _{\varepsilon \rightarrow 0}\left(\frac{\mathscr{F}(u+\varepsilon v)-\mathscr{F}(u)}{\varepsilon}, w\right)=-b\left(U(u), A^{-1 / 2} w, U^{\prime}(u) v\right)-b\left(U^{\prime}(u) v, A^{-1 / 2} w, U(u)\right),
$$

for all $u, v, w \in H$. This implies (3.46). 
Finally, we show that $\left|\mathscr{F}^{\prime}(u)\right|_{\mathcal{L}(H, H)}$ is uniformly bounded. By $(3.46)$ we estimate

$$
\begin{aligned}
\left(\mathscr{F}^{\prime}(u) v, w\right) & \leq\left|b\left(U(u), A^{-1 / 2} w, U^{\prime}(u) v\right)\right|+\left|b\left(U^{\prime}(u) v, A^{-1 / 2} w, U(u)\right)\right| \\
& \leq C|U(u)|_{L^{\infty}}|w|\left|U^{\prime}(u) v\right| \\
& \leq C|U(u)|_{H^{2}}|w|\left|U^{\prime}(u) v\right| \\
& \leq C L_{1}|w||v| \text { for all } w \in H,
\end{aligned}
$$

where we have used (3.35) and (3.37) to obtain the last inequality. It follows that

$$
\left|\mathscr{F}^{\prime}(u)\right|_{\mathcal{L}(H, H)} \leq C L_{1}, \text { for all } u \in H
$$

This completes the proof.

Next, we verify the spatial averaging condition (see Theorem 2.4 for the "prepared" equation (3.44) in $\mathbb{T}^{3}$.

Proposition 3.6. Let $\mathscr{F}$ be the operator defined by (3.43). Given $\delta>0$. There exist arbitrarily large $N \in \mathbb{N}$ and $k \in\left[\gamma \log \lambda_{N}, \lambda_{N}\right)$ for some constant $\gamma \in(0,1]$ independent of $N$, such that

$$
\left|R_{k, N} \mathscr{F}^{\prime}(u) R_{k, N} v\right| \leq \delta|v|
$$

for any $u, v \in H$.

Proof. Let $u=\left(u_{1}, u_{2}, u_{3}\right), v=\left(v_{1}, v_{2}, v_{3}\right), w=\left(w_{1}, w_{2}, w_{3}\right)$ be vectors in $H$. Then, due to (3.46), we have

$$
\begin{aligned}
& \left(R_{k, N} \mathscr{F}^{\prime}(u) R_{k, N} v, w\right)=\left(\mathscr{F}^{\prime}(u) R_{k, N} v, R_{k, N} w\right) \\
& =-b\left(U(u), A^{-1 / 2} R_{k, N} w, U^{\prime}(u) R_{k, N} v\right)-b\left(U^{\prime}(u) R_{k, N} v, A^{-1 / 2} R_{k, N} w, U(u)\right) .
\end{aligned}
$$

We denote by $U(u)_{n}$ the $n$-th component of the vector $U(u)$, and denote by $\left[U^{\prime}(u) v\right]_{n}$ the $n$-th component of the vector $U(u) v$, where $n=1,2,3$. By applying (3.5) we write

$$
\begin{aligned}
& b\left(U(u), A^{-1 / 2} R_{k, N} w, U^{\prime}(u) R_{k, N} v\right) \\
& =\sum_{m, n=1}^{3} \int_{\mathbb{T}^{3}}\left(U(u)_{m} R_{k, N}\left[U^{\prime}(u) v\right]_{n}\right)\left(R_{k, N} \partial_{m} A^{-1 / 2} w_{n}\right) d x \\
& =\sum_{m, n=1}^{3} \int_{\mathbb{T}^{3}} R_{k, N}\left(U(u)_{m} R_{k, N}\left[U^{\prime}(u) v\right]_{n}\right) \partial_{m} A^{-1 / 2} w_{n} d x .
\end{aligned}
$$

It follows that

$$
\left|b\left(U(u), A^{-1 / 2} R_{k, N} w, U^{\prime}(u) R_{k, N} v\right)\right| \leq|w| \sum_{m, n=1}^{3}\left|R_{k, N}\left(U(u)_{m} R_{k, N}\left[U^{\prime}(u) v\right]_{n}\right)\right| .
$$

The same estimate holds for the second term on the right-hand side of (3.54). Therefore,

$$
\left(R_{k, N} \mathscr{F}^{\prime}(u) R_{k, N} v, w\right) \leq 2|w| \sum_{m, n=1}^{3}\left|R_{k, N}\left(U(u)_{m} R_{k, N}\left[U^{\prime}(u) v\right]_{n}\right)\right| .
$$


In order to estimate the right-hand side of 3.55 , we let $\varphi, \psi \in L^{2}\left(\mathbb{T}^{3}\right)$ such that $\varphi$ and $\psi$ have mean value zero. Let $r>0$ and then denote

$$
\varphi_{>r}:=\sum_{|j|>r} \hat{\varphi}_{j} e^{i j \cdot x}, \quad \text { and } \quad \varphi_{<r}:=\sum_{1 \leq|j| \leq r} \hat{\varphi}_{j} e^{i j \cdot x} .
$$

Thus, $\varphi=\varphi_{>r}+\varphi_{<r}$. Then

$$
R_{k, N}\left(\varphi R_{k, N} \psi\right)=R_{k, N}\left(\varphi_{>r} R_{k, N} \psi\right)+R_{k, N}\left(\varphi_{<r} R_{k, N} \psi\right) .
$$

By Proposition 4.5 (see Appendix 4.1), there exist arbitrarily large $\lambda>0$ and $\kappa \in[\gamma \log \lambda, \lambda$ ) for some $\gamma>0$ independent of the choice of $\lambda$, satisfying: whenever $|n|^{2},|l|^{2} \in[\lambda-\kappa, \lambda+\kappa]$ with distinct $n$ and $l \in \mathbb{Z}^{3}$ one has $|n-l|>r$. Without loss of generality, we assume $\gamma \in(0,1]$. Therefore, due to Weyl's law $\lambda_{N} \sim C N^{\frac{2}{3}}$, there exist arbitrarily large $\lambda_{N}>0$ and $k \in\left[\gamma \log \lambda_{N}, \lambda_{N}\right)$ satisfying: whenever $|n|^{2},|l|^{2} \in\left[\lambda_{N}-k, \lambda_{N}+k\right]$ with distinct $n$ and $l \in \mathbb{Z}^{3}$ one has $|n-l|>r$. It follows that

$$
R_{k, N}\left(\varphi_{<r} R_{k, N} \psi\right)=\sum_{\lambda_{N}-k \leq|n|^{2} \leq \lambda_{N}+k}\left(\sum_{\substack{\lambda_{N}-k \leq|l|^{2} \leq \lambda_{N}+k \\ 1 \leq|n-l| \leq r}} \hat{\varphi}_{n-l} \hat{\psi}_{l}\right) e^{i n \cdot x}=0 .
$$

Due to (3.56) and (3.57), for the chosen $N$ and $k$, we have

$$
R_{k, N}\left(\varphi R_{k, N} \psi\right)=R_{k, N}\left(\varphi_{>r} R_{k, N} \psi\right) .
$$

Consequently, by assuming $\varphi \in H^{2}$ with zero mean and using a basic interpolation inequality, we obtain

$$
\left|R_{k, N}\left(\varphi R_{k, N} \psi\right)\right| \leq\left|\varphi_{>r} R_{k, N} \psi\right| \leq\left|\varphi_{>r}\right|_{L^{\infty}}|\psi| \leq\left|\varphi_{>r}\right|^{\frac{1}{4}}|\varphi|_{H^{2}}^{\frac{3}{4}}|\psi| .
$$

Notice that

$$
\left|\varphi_{>r}\right|^{2}=\sum_{|j|>r}\left|\hat{\varphi}_{j}\right|^{2}=\sum_{|j|>r} \frac{1}{|j|^{4}}|j|^{4}\left|\hat{\varphi}_{j}\right|^{2} \leq \frac{1}{r^{4}}|\varphi|_{H^{2}}^{2} .
$$

By (3.58) and (3.59), it follows that

$$
\left|R_{k, N}\left(\varphi R_{k, N} \psi\right)\right| \leq \frac{1}{\sqrt{r}}|\varphi|_{H^{2}}|\psi| .
$$

By (3.55) and (3.60), one has

$$
\left(R_{k, N} \mathscr{F}^{\prime}(u) R_{k, N} v, w\right) \leq \frac{2|w|}{\sqrt{r}} \sum_{m, n=1}^{3}\left|U(u)_{m}\right|_{H^{2}}\left|\left[U^{\prime}(u) v\right]_{n}\right| .
$$

By (3.35) and (3.37) in Lemma 3.4, we see that $|U(u)|_{H^{2}} \leq C$ for all $u \in H$ and $\left|U^{\prime}(u) v\right| \leq$ $L_{1}|v|$. Therefore, we derive from (3.61) that

$$
\left(R_{k, N} \mathscr{F}^{\prime}(u) R_{k, N} v, w\right) \leq \frac{C L_{1}}{\sqrt{r}}|v||w|, \text { for all } u, v, w \in H .
$$

This implies that

$$
\left|R_{k, N} \mathscr{F}^{\prime}(u) R_{k, N} v\right| \leq \frac{C L_{1}}{\sqrt{r}}|v| \leq \delta|v|, \quad \text { for all } u, v, w \in H
$$


by choosing $r$ sufficiently large. Notice that the value of $r$ does not depend on $N$ and $k$. This completes the proof.

3.4. Existence of an inertial manifold. Finally, we state and prove the main result of the manuscript: the existence of inertial manifolds for the hyperviscous Navier-Stokes equations 1.1 for the critical case $\beta=3 / 2$. Recall that, if $f \in H^{1}$, all solutions of $u_{t}+A^{3 / 2} u+B(u, u)=f$ enter an absorbing ball $\mathscr{B}$ in $H^{4}$ for large time, which coincide with solutions of the "prepared" equation $(3.62)$ in $\mathscr{B}$.

Theorem 3.7. Consider the "prepared" equation for the incompressible hyperviscous NavierStokes equations on $\mathbb{T}^{d}, d=2$ or 3 , namely,

$$
u_{t}+A^{3 / 2} u+B(U(u), U(u))=f,
$$

for $f \in H^{1}$. The equation (3.62) possesses an inertial manifold in $H$ in the sense of Definition 2.1.

Proof. First, we write 3.62$)$ in the form

$$
u_{t}+A^{3 / 2} u+A^{1 / 2} \mathscr{F}(u)=f,
$$

where $\mathscr{F}(u)=A^{-1 / 2} B(U(u), U(u)$ ). By Proposition 3.5 (whose statement is valid in both two and three dimensions), $\mathscr{F}$ is Gateaux differentiable from $H$ to $H$ such that $\left|\mathscr{F}^{\prime}(u)\right|_{\mathcal{L}(H, H)} \leq L$ for all $u \in H$. Also $|\mathscr{F}(u)|_{H^{2}} \leq C$ for all $u \in H$, for some constant $C>0$. Let $\left\{\lambda_{n}=|j|^{2}: j \in \mathbb{Z}^{d} \backslash\{0\}\right\}, d=2$ or 3 , such that $0<\lambda_{1} \leq \lambda_{2} \leq \lambda_{3} \leq \cdots$, correspond to the eigenvectors $\left\{e^{i j \cdot x}: j \in \mathbb{Z}^{d} \backslash\{0\}\right\}$ of $A$ in $H$.

Case 1: If there exists a sufficiently large spectral gap, namely, there exists $N \in \mathbb{N}$ such that $\lambda_{N+1}-\lambda_{N} \geq 2 L$, then by Theorem 2.2 , there exists an $N$-dimensional inertial manifold for (3.62). In particular, on the two-dimensional periodic domain $\mathbb{T}^{2}$, an arbitrary large gap for eigenvalues of $A=-\Delta$ is guaranteed by Theorem 4.2 (see the Appendix), due to Richards [29].

Case 2: In three dimensions, there does not necessarily exist a sufficiently large spectral gap. But, by Proposition 3.6, there exist $N \in \mathbb{N}$ and $k \in\left[\gamma \log \lambda_{N}, \lambda_{N}\right)$ for some $\gamma \in(0,1]$ such that $\lambda_{N} \geq e^{40 L^{2} / \gamma}$ and $1 \leq \lambda_{N+1}-\lambda_{N} \leq 2 L$, and

$$
\left|R_{k, N} \mathscr{F}^{\prime}(u) R_{k, N} v\right| \leq \delta|v|, \text { for all } u, v \in H,
$$

for some $\delta \leq \frac{1}{50}$. Consequently, the spatial averaging condition 2.6 is fulfilled, so we conclude from Corollary 2.8 that equation 3.62 possesses an $N$-dimensional inertial manifold in $H$.

\section{Appendix}

In this appendix, we include a series of well-known theoretical results (see Appendix 4.1) and then provide the complete proof of Theorem 2.7 (see Appendix 4.2). We also provide a proof of the strong cone property when the optimal spectral gap condition is satisfied (see Appendix 4.3), and a proof of the global well-posedness of weak solutions and the existence of global attractors for the hyperviscous Navier-Stokes equations (1.1) when $\beta \geq 5 / 4$ in three-dimensional domains (see Appendix 4.4). 
4.1. Supporting results. First let us recall the uniform Gronwall inequality.

Lemma 4.1 (Temam [33]). Let $g$, $h$ and y be nonnegative locally integrable functions defined for $t \geq t_{0}$. Suppose

$$
\frac{d y}{d t} \leq g y+h, \text { for } t \geq t_{0}
$$

Then, it holds

$$
y(t+1) \leq e^{\int_{t}^{t+1} g(\tau) d \tau}\left(\int_{t}^{t+1} y(\tau) d \tau+\int_{t}^{t+1} h(\tau) d \tau\right), \text { for } t \geq t_{0} .
$$

Next we state a few number theoretic results which are related to the construction of an inertial manifold.

Theorem 4.2 (Richards [29]). The sequence $\left\{s_{n}=k_{1}^{2}+k_{2}^{2}: k_{1}, k_{2} \in \mathbb{Z}\right.$ and $\left.s_{n+1} \geq s_{n}\right\}$ satisfies

$$
\limsup _{n \rightarrow \infty} \frac{s_{n+1}-s_{n}}{\log s_{n}} \geq \delta
$$

for some $\delta>0$.

Theorem 4.3 (Mallet-Paret and Sell [26]). Let $D \subset \mathbb{Z}$ be a finite nonempty set of integers with the property that $\prod_{d \in A}$ d is not a perfect square whenever $A \subset D$ has odd cardinality. There exist arbitrarily large $m \in \mathbb{N}$ and $h \geq C \log m$ for some constant $C>0$ independent of $m$, satisfying: if $T$ is any quadratic form

$$
T\left(k_{1}, k_{2}\right)=a k_{1}^{2}+b k_{1} k_{2}+c k_{2}^{2}, \quad a, b, c \in \mathbb{Z},
$$

with discriminant $d=b^{2}-4 a c \in D$, then

$$
T\left(k_{1}, k_{2}\right) \notin[m, m+h] \text { for each } k_{1}, k_{2} \in \mathbb{Z} \text {. }
$$

Proof. Theorem 4.3 has been proved by Mallet-Paret and Sell in [26]. The estimate of the size of the gap $h$, i.e., $h \geq C \log m$ has been claimed in [26] without a detailed argument. For the sake of completion, we present the proof for the estimate $h \geq C \log m$ as follows. The idea is from the proof of Theorem 4.2 by Richards in [29]. Indeed, since we consider the discriminant $d=b^{2}-4 a c \in D$, without loss of generality, one may assume that every element $d$ in $D$ satisfying $d \equiv 0$ or $1(\bmod 4)$. It has been proved in [26] that there exists $r \neq 0$ such that

$$
\operatorname{gcd}(d, r)=1 \text { and }\left(\frac{d}{r}\right)=-1, \text { for each } d \in D,
$$

where $\left(\frac{d}{r}\right)$ is the Jacobi symbol. Define $\delta:=\operatorname{lcm}\{|d|: d \in D\}$. By 4.1), one has $\operatorname{gcd}(\delta, r)=1$. Let $h>0$ and set

$$
A:=\sup _{0 \leq j \leq h}|r+\delta j|
$$

Define $P$ be the product

$$
P:=\prod p^{1+\alpha}
$$


where the product is taken over all primes $p$ with

$$
p \nmid \delta \text { and } p^{\alpha} \leq A<p^{1+\alpha} \text { for some integer } \alpha>0 .
$$

$\operatorname{Recall} \operatorname{gcd}(\delta, r)=1$. Set the integer $m \in[1, P]$ satisfying

$$
\delta m \equiv r(\bmod P) .
$$

It has been shown in [26] that $T\left(k_{1}, k_{2}\right) \notin[m, m+h]$ for any $k_{1}, k_{2} \in \mathbb{Z}$. We argue that $h \geq C \log m$. Indeed, the number of primes $p \leq A$ is asymptotic to $\frac{A}{\log A}$. By 4.3 , we obtain that

$$
P \leq A^{\frac{2(1+\varepsilon) A}{\log A}}=e^{2(1+\varepsilon) A}, \text { for some } \varepsilon>0
$$

This implies that

$$
A \geq \frac{\log P}{2(1+\varepsilon)} \geq \frac{\log m}{2(1+\varepsilon)}
$$

since $P \geq m$. Moreover, since $r$ and $\delta$ are fixed integers, then $h$ and $A$ can be chosen arbitrarily large such that 4.2 is valid and

$$
h \geq \frac{A}{2 \delta} .
$$

In view of (4.6) and 4.7), we conclude that

$$
h \geq \frac{\log m}{4 \delta(1+\varepsilon)} .
$$

This demonstrates the size of the gap stated in Theorem 4.3 .

The following result is a corollary of Theorem 4.3 .

Corollary 4.4 (Mallet-Paret and Sell [26]). Let $\mathscr{T}$ be a finite collection of functions $T$ of the form

$$
T\left(k_{1}, k_{2}\right)=a k_{1}^{2}+b k_{1} k_{2}+c k_{2}^{2}+s k_{1}+t k_{2}+u,
$$

with rational coefficients and negative discriminant, i.e.,

$$
a, b, c, s, t, u \in \mathbb{Q}, \quad b^{2}-4 a c<0 .
$$

Then, there exist arbitrarily large $m \in \mathbb{N}$ and $h \geq C \log m$ for some constant $C>0$ independent of $m$, such that

$$
T\left(k_{1}, k_{2}\right) \notin[m, m+h] \text { for each } T \in \mathscr{T} \text { and } k_{1}, k_{2} \in \mathbb{Z} .
$$

Corollary 4.4 implies the following proposition.

Proposition 4.5 (Mallet-Paret and Sell [26]). Let $r>0$. There exist arbitrarily large $\lambda>0$ and $\kappa>C \log \lambda$ for some constant $C>0$ independent of $\lambda$, satisfying: whenever $|n|^{2},|l|^{2} \in[\lambda-\kappa, \lambda+\kappa]$ with distinct $n$ and $l \in \mathbb{Z}^{3}$ one has $|n-l|>r$. 
4.2. Proof of Theorem 2.7. It is standard that the cone invariance property and the decay property together imply the existence of an inertial manifold in $H$ for the model problem (2.5). We present the proof of Theorem 2.7 for the sake of completion.

Proof of Theorem 2.7. We adopt the proof by Zelik [35] (see also Kostianko and Zelik [25]). The argument can be split into four steps.

Step 1: We aim to prove that the boundary-value problem

$$
\left\{\begin{array}{l}
u_{t}+A^{3 / 2} u+A^{1 / 2} F(u)=f \\
P_{N} u(0)=u_{0}, \quad Q_{N} u(-T)=0
\end{array}\right.
$$

has a unique solution for any $T>0$ and $u_{0} \in P_{N} H$.

In fact, let us denote by $S(t)$ the semigroup induced by 2.5 and consider the map $G_{T}: P_{N} H \rightarrow P_{N} H$ given by

$$
G_{T}(\varphi)=P_{N} S(T) \varphi, \quad \text { for } \varphi \in P_{N} H .
$$

First notice that $G_{T}$ is Lipschitz continuous. To see it, we let $\varphi_{1}$ and $\varphi_{2} \in P_{N} H$. Set $u_{1}(t)=S(t) \varphi_{1}$ and $u_{2}(t)=S(t) \varphi_{2}$. Due to 2.40 , one has

$$
\frac{d}{d t}\left|u_{1}(t)-u_{2}(t)\right|^{2} \leq L^{2}\left|u_{1}(t)-u_{2}(t)\right|^{2}, \quad \text { for all } t \geq 0
$$

Therefore,

$$
\left|u_{1}(t)-u_{2}(t)\right|^{2} \leq e^{t L^{2}}\left|\varphi_{1}-\varphi_{2}\right|^{2}, \quad \text { for all } t \geq 0
$$

It follows that

$$
\left|G_{T}\left(\varphi_{1}\right)-G_{T}\left(\varphi_{2}\right)\right|^{2} \leq e^{T L^{2}}\left|\varphi_{1}-\varphi_{2}\right|^{2}, \quad \text { for any } \varphi_{1}, \varphi_{2} \in P_{N} H
$$

This shows that $G_{T}: P_{N} H \rightarrow P_{N} H$ is Lipschitz continuous.

Next we shall justify that the map $G_{T}$ is injective. Put $v(t)=u_{1}(t)-u_{2}(t)$. Also set $p(t)=$ $P_{N} v(t)$ and $q(t)=Q_{N} v(t)$. Taking the inner product of $v_{t}+A^{3 / 2} v+A^{1 / 2}\left(F\left(u_{1}\right)-F\left(u_{2}\right)\right)=0$ with $p$ yields $\frac{1}{2} \frac{d}{d t}|p|^{2}+\left|A^{3 / 4} p\right|^{2}+\left(F\left(u_{1}\right)-F\left(u_{2}\right), A^{1 / 2} p\right)=0$, which implies

$$
\frac{1}{2} \frac{d}{d t}|p|^{2}+\lambda_{N}^{3 / 2}|p|^{2}+L \lambda_{N}^{1 / 2}|p||v| \geq 0,
$$

where we have used the Lipschitz continuity $\left|F\left(u_{1}\right)-F\left(u_{2}\right)\right| \leq L\left|u_{1}-u_{2}\right|=L|v|$.

Since $v(0)=\varphi_{1}-\varphi_{2} \in P_{N} H$, by the cone invariance property, $|q(t)| \leq|p(t)|$, for all $t \geq 0$. Thus $|v(t)| \leq 2|p(t)|$, for all $t \geq 0$. Then, due to 4.10), one has

$$
\frac{d}{d t}|p|^{2}+2\left(\lambda_{N}^{3 / 2}+2 L \lambda_{N}^{1 / 2}\right)|p|^{2} \geq 0, \quad \text { for all } t \geq 0
$$

As a result, $|p(0)| \leq e^{\left(\lambda_{N}^{3 / 2}+2 L \lambda_{N}^{1 / 2}\right) t}|p(t)|$ for all $t \geq 0$. Consequently, we have

$$
\left|\varphi_{1}-\varphi_{2}\right| \leq e^{\left(\lambda_{N}^{3 / 2}+2 L \lambda_{N}^{1 / 2}\right) T}\left|G_{T}\left(\varphi_{1}\right)-G_{T}\left(\varphi_{2}\right)\right| .
$$

This implies that $G_{T}: P_{N} H \rightarrow P_{N} H$ is injective, and $G_{T}^{-1}$ is Lipschitz continuous on its domain.

Since $P_{N} H$ is finite dimensional and $G_{T}$ is continuous and injective, by using the theorem of invariance of domain [5], we conclude that $G_{T}$ is a homeomorphism from $P_{N} H$ to an open set $G_{T}\left(P_{N} H\right)$. Due to 4.11), we see that the open set $G_{T}\left(P_{N} H\right)$ has no boundary. For, if $\psi_{0}$ 
is a point on the boundary of the open set $G_{T}\left(P_{N} H\right)$, then there exists a sequence $\left\{\psi_{n}\right\} \subset$ $G_{T}\left(P_{N} H\right)$ such that $\psi_{n} \rightarrow \psi_{0}$, and by 4.11), $G_{T}^{-1} \psi_{n}$ is a Cauchy sequence and has a limit point $\phi_{0}$ in $P_{N} H$, then $G_{T}\left(\phi_{0}\right)=\psi_{0}$ by the continuity of $G_{T}$, which contracts the assumption that $\psi_{0} \notin G_{T}\left(P_{N} H\right)$. As a result, $G_{T}\left(P_{N} H\right)=P_{N} H$, i.e., $G_{T}$ is a homeomorphism from $P_{N} H$ to $P_{N} H$. Therefore, $G_{T}(\varphi)=u_{0}$ is uniquely solvable for all $u_{0} \in P_{N} H$. It follows that $u(t)=S(t+T) G_{T}^{-1}\left(u_{0}\right)$ is the unique solution of the boundary-value problem 4.9 .

Step 2: Given $u_{0} \in P_{N} H$. Let $u_{T}(t)$ be the solution of the boundary-value problem $(4.9)$. We aim to prove that the limit

$$
\tilde{u}(t)=\lim _{T \rightarrow \infty} u_{T}(t)
$$

exists for all $t \leq 0$ and is a backward solution of the problem $u_{t}+A^{3 / 2} u+A^{1 / 2} F(u)=f$ with $P_{N} u(0)=u_{0}$.

Indeed, let $T_{2}>T_{1}>0$, and set $v(t)=u_{T_{1}}(t)-u_{T_{2}}(t)$, for $t \in\left[-T_{1}, 0\right]$. Put $p(t)=P_{N} v(t)$ and $q(t)=Q_{N} v(t)$. Notice that $p(0)=P_{N} u_{T_{1}}(0)-P_{N} u_{T_{2}}(0)=u_{0}-u_{0}=0$. Then by the cone invariance property, we have $|q(t)| \geq|p(t)|$ for all $t \in\left[-T_{1}, 0\right]$, and thus $2|q(t)| \geq|v(t)|$ for all $t \in\left[-T_{1}, 0\right]$. Then by the decay property, we obtain that

$$
|v(t)| \leq C e^{-\alpha\left(t+T_{1}\right)}\left|v\left(-T_{1}\right)\right| \leq 2 C e^{-\alpha\left(t+T_{1}\right)}\left|q\left(-T_{1}\right)\right|=2 C e^{-\alpha\left(t+T_{1}\right)}\left|Q_{N} u_{T_{2}}\left(-T_{1}\right)\right|,
$$

for all $t \in\left[-T_{1}, 0\right]$, where the last equality is due to the fact that $Q_{N} u_{T_{1}}\left(-T_{1}\right)=0$. In order to find a uniform bound for $\left|Q_{N} u_{T_{2}}\left(-T_{1}\right)\right|$ for any $T_{2}>T_{1}>0$, we consider an arbitrary solution $u_{T}$ of problem 4.9 . One has

$$
\begin{aligned}
& \frac{1}{2} \frac{d}{d t}\left|Q_{N} u_{T}\right|^{2}+\left|A^{3 / 4} Q_{N} u_{T}\right|^{2} \leq\left|\left(F\left(u_{T}\right), A^{1 / 2} Q_{N} u_{T}\right)\right|+\left|\left(f, Q_{N} u_{T}\right)\right| \\
& \leq\left|A^{-1 / 4} F\left(u_{T}\right)\right|^{2}+\frac{1}{4}\left|A^{3 / 4} Q_{N} u_{T}\right|^{2}+\left|A^{-3 / 4} f\right|^{2}+\frac{1}{4}\left|A^{3 / 4} Q_{N} u_{T}\right|^{2}
\end{aligned}
$$

for $t \in[-T, 0]$. It follows that

$$
\frac{d}{d t}\left|Q_{N} u_{T}\right|^{2}+\left|A^{3 / 4} Q_{N} u_{T}\right|^{2} \leq 2\left|A^{-1 / 4} F\left(u_{T}\right)\right|^{2}+2\left|A^{-3 / 4} f\right|^{2} \leq C,
$$

for $t \in[-T, 0]$, due to the assumption that $\left|A^{-1 / 4} F(u)\right|$ is uniformly bounded for all $u \in H$.

As a result, we have

$$
\frac{d}{d t}\left|Q_{N} u_{T}\right|^{2}+\lambda_{N+1}^{3 / 2}\left|Q_{N} u_{T}\right|^{2} \leq C, \quad \text { for } t \in[-T, 0]
$$

Since $u_{T}$ is a solution of 4.9), we know that $Q_{N} u_{T}(-T)=0$, then by 4.14), one has

$$
\left|Q_{N} u_{T}(t)\right|^{2} \leq C
$$

for some constant $C>0$ and all $t \in[-T, 0]$ and for any $T>0$. It follows that

$$
\left|Q_{N} u_{T_{2}}\left(T_{1}\right)\right|^{2} \leq C \text {. }
$$

Therefore, by (4.13), we obtain that

$$
\left|u_{T_{1}}(t)-u_{T_{2}}(t)\right|=|v(t)| \leq C e^{-\alpha\left(t+T_{1}\right)}, \quad \text { for all } t \in\left[-T_{1}, 0\right],
$$

for any $T_{2}>T_{1}>0$. Consequently, for any $t^{*}>0$, as $T_{1}, T_{2} \rightarrow \infty$, one has

$$
\left|u_{T_{1}}(t)-u_{T_{2}}(t)\right| \rightarrow 0, \quad \text { uniformly on }\left[-t^{*}, 0\right] \text {. }
$$


This implies that $u_{T}$ is a Cauchy sequence in $C\left(\left[-t^{*}, 0\right] ; H\right)$ for any $t^{*}>0$. Hence, $\tilde{u}=$ $\lim _{T \rightarrow \infty} u_{T}$ exists in $C\left(\left[-t^{*}, 0\right] ; H\right)$ for any $t^{*}>0$, where $u_{T}$ is the solution of $(4.9)$. This shows that $\tilde{u}(t)$ is a backward solution of $u_{t}+A^{3 / 2} u+A^{1 / 2} F(u)=f$ with $P_{N} u(0)=u_{0}$ and it has a unique extension $\tilde{u}$ which solves $u_{t}+A^{3 / 2} u+A^{1 / 2} F(u)=f$ for all $t \in \mathbb{R}$. Also, we remark that, due to 4.15$),\left|Q_{N} \tilde{u}(t)\right|$ is uniformly bounded for all $t \in \mathbb{R}$.

Step 3: Define the set $\mathcal{A}$ as the set of all solutions $\tilde{u}(t), t \in \mathbb{R}$, of (2.5) constructed in Step 2 using the limiting process $(4.12)$. By the construction, the set $\mathcal{A}$ is invariant in time, i.e,

$$
T(\tau) \mathcal{A}=\mathcal{A}, \quad \text { where }(T(\tau) \tilde{u})(t)=\tilde{u}(t+\tau), \quad \tau \in \mathbb{R} .
$$

Also, for two trajectories $\tilde{u}_{1}$ and $\tilde{u}_{2} \in \mathcal{A}$, we claim that

$$
\left|Q_{N}\left(\tilde{u}_{1}(t)-\tilde{u}_{2}(t)\right)\right| \leq\left|P_{N}\left(\tilde{u}_{1}(t)-\tilde{u}_{2}(t)\right)\right|, \text { for all } t \in \mathbb{R} .
$$

Indeed, by the limiting process 4.12 , there exist $u_{0}^{1}$ and $u_{0}^{2} \in P_{N} H$ such that $\lim _{T \rightarrow \infty} u_{T, u_{0}^{1}}=$ $\tilde{u}_{1}$ and $\lim _{T \rightarrow \infty} u_{T, u_{0}^{2}}=\tilde{u}_{2}$, where $u_{T, u_{0}^{j}}, j=1,2$, are solutions of 2.5 with the boundary condition $P_{N} u(0)=u_{0}^{j}, Q_{N} u(-T)=0$. Since $Q_{N} u_{T, u_{0}^{1}}(-T)=Q_{N} u_{T, u_{0}^{2}}(-T)=0$, then by the cone invariance property, we obtain that

$$
\left|Q_{N}\left(u_{T, u_{0}^{1}}(t)-u_{T, u_{0}^{2}}(t)\right)\right| \leq\left|P_{N}\left(u_{T, u_{0}^{1}}(t)-u_{T, u_{0}^{2}}(t)\right)\right|, \quad \text { for all } t \geq-T .
$$

By passing to the limit on 4.17) as $T \rightarrow \infty$, we obtain (4.16).

We define the map $\Phi: P_{N} H \rightarrow Q_{N} H$ by

$$
\Phi\left(u_{0}\right)=Q_{N} \tilde{u}(0) \text { where } \tilde{u} \in \mathcal{A} \text { with } P_{N} \tilde{u}(0)=u_{0} .
$$

Due to 4.16), we have

$$
\left|Q_{N}\left(\tilde{u}_{1}(0)-\tilde{u}_{2}(0)\right)\right| \leq\left|P_{N}\left(\tilde{u}_{1}(0)-\tilde{u}_{2}(0)\right)\right| .
$$

That is,

$$
\left|\Phi\left(u_{0}^{1}\right)-\Phi\left(u_{0}^{2}\right)\right| \leq\left|u_{0}^{1}-u_{0}^{2}\right|, \quad \text { for any } u_{0}^{1}, u_{0}^{2} \in H .
$$

This shows that $\Phi: P_{N} H \rightarrow Q_{N} H$ is Lipschitz continuous with Lipschitz constant 1.

Now we construct the Lipschitz manifold of dimension $N$ as

$$
\mathcal{M}:=\left\{u_{0}+\Phi\left(u_{0}\right): u_{0} \in P_{N} H\right\} .
$$

The invariance of $\mathcal{A}$ implies the invariance of $\mathcal{M}$ with respect to the semigroup $S(t)$.

Step 4: We aim to show that any trajectory of the solution of 2.5) approaches the finitedimensional Lipschitz manifold $\mathcal{M}$ exponentially fast. Indeed, let $u(t), t \geq 0$, be a solution of (2.5) such that its trajectory does not belong to $\mathcal{M}$. For each $T>0$, there exists $\tilde{u}_{T} \in \mathcal{A}$ such that

$$
P_{N} u(T)=P_{N} \tilde{u}_{T}(T) .
$$

Then by the cone invariance property, we have

$$
\left|P_{N}\left(u(t)-\tilde{u}_{T}(t)\right)\right|<\left|Q_{N}\left(u(t)-\tilde{u}_{T}(t)\right)\right|, \text { for } t \in[0, T],
$$

and by the decay property, we have

$$
\left|u(t)-\tilde{u}_{T}(t)\right| \leq C e^{-\alpha t}\left|u(0)-\tilde{u}_{T}(0)\right|, \text { for } t \in[0, T] .
$$

Recall in Step 2, we have shown that, for any $\tilde{u} \in \mathcal{A},\left|Q_{N} \tilde{u}(t)\right|$ is uniformly bounded in $\mathbb{R}$. Hence, we have that $\left|Q_{N} \tilde{u}_{T}(0)\right|$ is uniformly bounded for all $T>0$. Also, due to (4.18), 
$\left|P_{N}\left(u(0)-\tilde{u}_{T}(0)\right)\right|<\left|Q_{N}\left(u(0)-\tilde{u}_{T}(0)\right)\right|$. As a result, $\left|\tilde{u}_{T}(0)\right|$ is uniformly bounded for all $T>0$. Since the Lipschitz manifold $\mathcal{M}$ is finite dimensional and $\tilde{u}_{T}(0) \in \mathcal{M}$, there exists a sequence $\tilde{u}_{T_{j}}(0) \rightarrow \tilde{u}(0) \in \mathcal{M}$ as $T_{j} \rightarrow \infty$ and the corresponding trajectory $\tilde{u}(t) \in \mathcal{A}$. Since $\tilde{u}_{T_{j}}$ and $\tilde{u}$ are both solutions of $(2.5)$, due to 2.40$)$, we obtain that

$$
\left|\tilde{u}_{T_{j}}(t)-\tilde{u}(t)\right|^{2} \leq e^{L^{2} t}\left|\tilde{u}_{T_{j}}(0)-\tilde{u}(0)\right|^{2}, \text { for all } t \geq 0 .
$$

This implies that $\left|\tilde{u}_{T_{j}}(t)-\tilde{u}(t)\right| \rightarrow 0$ as $T_{j} \rightarrow \infty$ for each $t \geq 0$. Therefore, by virtue of (4.19), we conclude that

$$
|u(t)-\tilde{u}(t)| \leq C e^{-\alpha t}|u(0)-\tilde{u}(0)|, \text { for } t \geq 0 .
$$

This shows that any trajectory $u(t)$ outside the inertial manifold $\mathcal{M}$ exponentially approaches a trajectory $\tilde{u}(t)$ in $\mathcal{M}$.

4.3. Derivation of the cone property under the optimal spectral gap condition. For the self-containedness of the paper, we derive a strong cone property for the model (2.1) with the assumption of the sharp spectral gap condition (2.4).

Proposition 4.6. Let $u_{1}$ and $u_{2}$ be two solutions of (2.1). Set $v=u_{1}-u_{2}$. Denote $p=P_{N} v$ and $q=Q_{N} v$. Define $\mathcal{V}(t)=|q|_{H^{-1 / 2}}^{2}-|p|_{H^{-1 / 2}}^{2}$. Assume $F: H \rightarrow H$ is globally Lipschitz continuous with Lipschitz constant L. Suppose the spectral gap condition (2.4) is satisfied. Then, the following strong cone property holds:

$$
\frac{1}{2} \frac{d}{d t} \mathcal{V}(t)+\alpha \mathcal{V}(t) \leq-\mu|v(t)|^{2}, \text { for all } t \geq 0,
$$

where $\alpha=\frac{\lambda_{N}^{\beta} \lambda_{N+1}^{1 / 2}+\lambda_{N+1}^{\beta} \lambda_{N}^{1 / 2}}{\lambda_{N+1}^{1 / 2}+\lambda_{N}^{1 / 2}}$ and $\mu=\frac{\lambda_{N+1}^{\beta}-\lambda_{N}^{\beta}}{\lambda_{N+1}^{1 / 2}+\lambda_{N}^{1 / 2}}-L>0$.

Proof. Let $u_{1}$ and $u_{2}$ be two solutions of (2.1) and set $v=u_{1}-u_{2}$, then one has

$$
v_{t}+A^{\beta} v+A^{1 / 2}\left[F\left(u_{1}\right)-F\left(u_{2}\right)\right]=0,
$$

where $\beta \geq 3 / 2$. Set $p=P_{N} v$ and $q=Q_{N} v$. Take the scalar product of 4.21) with $A^{-1 / 2} p$ and $A^{-1 / 2} q$ respectively:

$$
\left\{\begin{array}{l}
\frac{1}{2} \frac{d}{d t}|p|_{H^{-1 / 2}}^{2}+\left|A^{\beta / 2} p\right|_{H^{-1 / 2}}^{2}+\left(F\left(u_{1}\right)-F\left(u_{2}\right), p\right)=0 \\
\frac{1}{2} \frac{d}{d t}|q|_{H^{-1 / 2}}^{2}+\left|A^{\beta / 2} q\right|_{H^{-1 / 2}}^{2}+\left(F\left(u_{1}\right)-F\left(u_{2}\right), q\right)=0 .
\end{array}\right.
$$

Define $\mathcal{V}(t)=|q|_{H^{-1 / 2}}^{2}-|p|_{H^{-1 / 2}}^{2}$. Subtracting the two equations in 4.22 yields

$$
\frac{1}{2} \frac{d}{d t} \mathcal{V}(t)+\left(\left|A^{\beta / 2} q\right|_{H^{-1 / 2}}^{2}-\left|A^{\beta / 2} p\right|_{H^{-1 / 2}}^{2}\right)=\left(F\left(u_{1}\right)-F\left(u_{2}\right), p-q\right) \leq L|v|^{2},
$$

due to the assumption that $F: H \rightarrow H$ is globally Lipschitz with Lipschitz constant $L$. It follows that

$$
\frac{1}{2} \frac{d}{d t} \mathcal{V}(t)+\alpha \mathcal{V}(t) \leq-\left(\left|A^{\beta / 2} q\right|_{H^{-1 / 2}}^{2}-\alpha|q|_{H^{-1 / 2}}^{2}\right)-\left(\alpha|p|_{H^{-1 / 2}}^{2}-\left|A^{\beta / 2} p\right|_{H^{-1 / 2}}^{2}\right)+L|v|^{2}
$$


Since $\alpha=\frac{\lambda_{N}^{\beta} \lambda_{N+1}^{1 / 2}+\lambda_{N+1}^{\beta} \lambda_{N}^{1 / 2}}{\lambda_{N+1}^{1 / 2}+\lambda_{N}^{1 / 2}}$, we calculate

$$
\alpha|p|_{H^{-1 / 2}}^{2}-\left|A^{\beta / 2} p\right|_{H^{-1 / 2}}^{2}=\sum_{j=1}^{N} \frac{\alpha-\lambda_{j}^{\beta}}{\lambda_{j}^{1 / 2}}\left|v_{j}\right|^{2} \geq \frac{\alpha-\lambda_{N}^{\beta}}{\lambda_{N}^{1 / 2}}|p|^{2}=\frac{\lambda_{N+1}^{\beta}-\lambda_{N}^{\beta}}{\lambda_{N+1}^{1 / 2}+\lambda_{N}^{1 / 2}}|p|^{2} .
$$

Also,

$$
\left|A^{\beta / 2} q\right|_{H^{-1 / 2}}^{2}-\alpha|q|_{H^{-1 / 2}}^{2}=\sum_{j=N+1}^{\infty} \frac{\lambda_{j}^{\beta}-\alpha}{\lambda_{j}^{1 / 2}}\left|v_{j}\right|^{2} \geq \frac{\lambda_{N+1}^{\beta}-\alpha}{\lambda_{N+1}^{1 / 2}}|q|^{2}=\frac{\lambda_{N+1}^{\beta}-\lambda_{N}^{\beta}}{\lambda_{N+1}^{1 / 2}+\lambda_{N}^{1 / 2}}|q|^{2} .
$$

Substituting 4.24) and 4.25 into 4.23, we obtain

$$
\frac{1}{2} \frac{d}{d t} \mathcal{V}(t)+\alpha \mathcal{V}(t) \geq-\left(\frac{\lambda_{N+1}^{\beta}-\lambda_{N}^{\beta}}{\lambda_{N+1}^{1 / 2}+\lambda_{N}^{1 / 2}}-L\right)|v|^{2}
$$

where $\mu=\frac{\lambda_{N+1}^{\beta}-\lambda_{N}^{\beta}}{\lambda_{N+1}^{1 / 2}+\lambda_{N}^{1 / 2}}-L>0$ owing to the spectral gap condition 2.4 .

By adopting the proof of Proposition 2.5 and using the estimates $\frac{d}{d t}|v(t)|^{2} \leq L^{2}|v(t)|^{2}$ as well as $\frac{d}{d t}|v(t)|_{H^{-1 / 2}}^{2} \leq L|v(t)|^{2}$, it can be readily shown that the strong cone property 4.20 implies

- Cone invariance property: If $\mathcal{V}(0) \leq 0$, then $\mathcal{V}(t) \leq 0$ for all $t \geq 0$;

- Decay property: If $\mathcal{V}(T)>0$ for some $T>0$, then

$$
|v(t)|^{2} \leq C e^{-\alpha t}|v(0)|^{2} \text { and }|v(t)|_{H^{-1 / 2}}^{2} \leq C e^{-\alpha t}|v(0)|_{H^{-1 / 2}}^{2} \quad \text { for all } t \in[0, T] \text {. }
$$

It is standard that the above cone invariance and the decay properties lead to the existence of an inertial manifold in $H^{-1 / 2}$, and thanks to the parabolic smoothing property, it immediately becomes an inertial manifold in the phase space $H$.

4.4. Global well-posedness and global attractors of equation (1.1). For the reader's convenience, we provide a brief analysis of the hyperviscous Navier-Stokes equations (1.1) in three dimensions when $\beta \geq 5 / 4$. For the sake of clarity, we consider the borderline case that $\beta=5 / 4$. Our analysis can readily be adapted to treat the general case $\beta \geq 5 / 4$. (The reader may also refer to [8, 22] for well-posedness and the existence of global attractors for some general families of problems including (1.1)). Without loss of generality, we assume $\nu=1$. So in the following analysis, we consider

$$
u_{t}+A^{5 / 4} u+B(u, u)=f \text { in } \mathbb{T}^{3},\left.u\right|_{t=0}=u_{0} .
$$

Theorem 4.7. Let $f \in H^{-5 / 4}$. Then for every $u_{0} \in H$, problem (4.27) possesses a unique weak solution such that $u \in C([0, T] ; H) \cap L^{2}\left(0, T ; H^{5 / 4}\right)$ with $u_{t} \in L^{2}\left(0, T ; H^{-5 / 4}\right)$ for an arbitrary time $T>0$. The weak solution depends continuously on the initial data.

Proof. We provide the following a priori estimates. By taking the inner product of (4.27) with $u$, it is easy to deduce that

$$
\frac{d}{d t}|u|^{2}+|u|_{H^{5 / 4}}^{2} \leq|f|_{H^{-5 / 4}}^{2}
$$


From 4.28 , we see that

$$
|u(t)|^{2} \leq e^{-t}\left|u_{0}\right|^{2}+|f|_{H^{-5 / 4}}^{2}\left(1-e^{-t}\right), \text { for } t \geq 0
$$

and

$$
\int_{0}^{t}|u|_{H^{5 / 4}}^{2} d \tau \leq\left|u_{0}\right|^{2}+t|f|_{H^{-5 / 4}}^{2}, \text { for } t \geq 0 .
$$

Using the Sobolev imbedding $H^{s} \hookrightarrow L^{\frac{6}{3-2 s}}$ in three dimensions for $s \in(0,3 / 2)$, we deduce

$$
(B(u, u), \phi)=b(u, u, \phi) \leq C|\phi|_{L^{12}}|\nabla u|_{L^{12 / 5}}|u| \leq C|\phi|_{H^{5 / 4}}|u|_{H^{5 / 4}}|u| .
$$

It follows that $|B(u, u)|_{H^{-5 / 4}} \leq C|u|_{H^{5 / 4}}|u|$. Then due to 4.29$)$ and (4.30), we have $B(u, u)$ is bounded in $L^{2}\left(0, T ; H^{-5 / 4}\right)$, and thus

$$
u_{t} \text { is bounded in } L^{2}\left(0, T ; H^{-5 / 4}\right) \text {. }
$$

On one hand, the existence of global weak solutions can be easily obtained by standard Galerkin method, using the a priori estimates (4.29)-(4.31) and a compactness argument. On the other hand, it is crucial to verify the uniqueness of weak solutions, which explains why $\beta=5 / 4$ is the borderline case for the well-posedness of 3D hyperviscous Navier-Stokes equations. To show the uniqueness, we take two solutions $u_{1}$ and $u_{2}$ of (4.27) and set $v=u_{1}-u_{2}$. Then, we have

$$
v_{t}+A^{5 / 4} v+B\left(u_{1}, v\right)+B\left(v, u_{2}\right)=0 .
$$

Take the $L^{2}$ inner product of 4.32 with $v$, then

$$
\begin{aligned}
& \frac{1}{2} \frac{d}{d t}|v|^{2}+|v|_{H^{5 / 4}}^{2} \leq\left|b\left(v, u_{2}, v\right)\right|=\left|b\left(v, v, u_{2}\right)\right| \\
& \leq C\left|u_{2}\right|_{L^{12}}|\nabla v|_{L^{12 / 5}}|v| \leq C\left|u_{2}\right|_{H^{5 / 4}}|v|_{H^{5 / 4}}|v| \leq \frac{1}{2}|v|_{H^{5 / 4}}^{2}+C\left|u_{2}\right|_{H^{5 / 4}}^{2}|v|^{2} .
\end{aligned}
$$

It follows that

$$
\frac{d}{d t}|v|^{2}+|v|_{H^{5 / 4}}^{2} \leq C\left|u_{2}\right|_{H^{5 / 4}}^{2}|v|^{2}
$$

Thanks to the Gronwall inequality, we obtain from 4.33 and 4.30 that

$$
|v(t)|^{2} \leq \exp \left(C \int_{0}^{t}\left|u_{2}\right|_{H^{5 / 4}}^{2} d \tau\right)|v(0)|^{2} \leq C \exp \left(\left|u_{2}(0)\right|^{2}+t|f|_{H^{-5 / 4}}^{2}\right)|v(0)|^{2},
$$

for $t \geq 0$. If $v(0)=u_{1}(0)-u_{2}(0)=0$, then (4.34) shows that $v(t)=u_{1}(t)-u_{2}(t)=0$ for all $t \geq 0$, so weak solutions are unique. The inequality (4.34) also implies the continuous dependence on initial data.

Theorem 4.8. Let $f \in H^{-1 / 4}$. Then the dynamics induced by (4.27) possesses a compact global attractor in the phase space $H$.

Proof. Estimate 4.29 implies that all trajectories of the dynamics induced by 4.27) enter an absorbing ball of radius $2|f|_{H^{-5 / 4}}$ in $H$. Namely,

$$
|u(t)| \leq 2|f|_{H^{-5 / 4}}, \text { for } t \geq t_{0} .
$$


To construct an absorbing ball in $H^{1}$, we take the inner product of 4.27) with $A u$,

$$
\begin{aligned}
& \frac{1}{2} \frac{d}{d t}|u|_{H^{1}}^{2}+|u|_{H^{9 / 4}}^{2} \leq|b(u, u, A u)|+|(f, A u)| \\
& \leq C|u|_{L^{12}}|A u|_{L^{12 / 5}}|u|_{H^{1}}+|u|_{H^{9 / 4}}|f|_{H^{-1 / 4}} \\
& \leq C|u|_{H^{5 / 4}}|u|_{H^{9 / 4}}|u|_{H^{1}}+\frac{1}{4}|u|_{H^{9 / 4}}^{2}+|f|_{H^{-1 / 4}}^{2} \leq C|u|_{H^{5 / 4}}^{2}|u|_{H^{1}}^{2}+\frac{1}{2}|u|_{H^{9 / 4}}^{2}+|f|_{H^{-1 / 4}}^{2} .
\end{aligned}
$$

Hence,

$$
\frac{d}{d t}|u|_{H^{1}}^{2}+|u|_{H^{9 / 4}}^{2} \leq C|u|_{H^{5 / 4}}^{2}|u|_{H^{1}}^{2}+2|f|_{H^{-1 / 4}}^{2}
$$

Notice that $\int_{t}^{t+1}|u|_{H^{5 / 4}}^{2} d \tau \leq|u(t)|^{2}+|f|_{H^{-5 / 4}}^{2} \leq 5|f|_{H^{-5 / 4}}^{2}$ for $t \geq t_{0}$ owing to 4.28 and (4.35). Therefore, by using the uniform Gronwall inequality on (4.36), we obtain

$$
|u(t)|_{H^{1}}^{2} \leq \rho^{2}:=\exp \left(C|f|_{H^{-5 / 4}}^{2}\right)\left(5|f|_{H^{-5 / 4}}^{2}+2|f|_{H^{-1 / 4}}^{2}\right), \text { for } t \geq t_{0}+1 \text {. }
$$

Estimate (4.37) shows that all trajectories of the dynamics enter an absorbing ball of the radius $\rho$ in $H^{1}$ for large time. Therefore, due to the standard theory of attractors (see [33]), the dynamics possesses a compact global attractor in the phase space $H$.

Acknowledgment. The authors are grateful to the reviewer for providing valuable comments and suggestions which further improved the quality of the paper.

\section{REFERENCES}

[1] M. Abu Hamed, Y. Guo, and E. S. Titi, Inertial manifolds for certain subgrid-scale $\alpha$-models of turbulence, SIAM J. Appl. Dyn. Syst. 14 (2015), 1308-1325.

[2] J. Avrin, Singular initial data and uniform global bounds for the hyper-viscous Navier-Stokes equations with periodic boundary conditions, J. Differential Equations 190 (2003), 330-351.

[3] J. Avrin, The asymptotic finite-dimensional character of a spectrally-hyperviscous model of $3 D$ turbulent flow, J. Dynam. Differential Equations 20 (2008), 479-518.

[4] V. Borue and S. A. Orszag, Local energy flux and subgrid-scale statistics in three-dimensional turbulence, J. Fluid Mech. 366 (1998), 1-31.

[5] L. E. J. Brouwer, Zur Invarianz des n-dimensionalen Gebiets, (German) Math. Ann. 72 (1912), 55-56.

[6] C. Basdevant, B. Legras, R. Sadourny, and M. Beland, A study of barotropic model flows: intermittency, waves, and predictability, J. Atmospheric Sci. 38 (1981), 2305-2320.

[7] G. L. Browning and H. O. Kreiss, Comparison of numerical methods for the calculation of twodimensional turbulence, Math. Comp. 52 (1989), 369-388.

[8] J. W. Cholewa and T. Dlotko, Fractional Navier-Stokes equations, Discrete Cont. Dyn. Syst. B, doi:10.3934/dcdsb.2017149.

[9] Y. Cao, E. Lunasin, and E. S. Titi, Global well-posedness of the three-dimensional viscous and inviscid simplified Bardina turbulence models, Commun. Math. Sci. 4 (2006), 823-848.

[10] S. Cerruto, C. Meneveau, and O. M. Knio, Spectral and hyper-eddy viscosity in high-Reynolds-number turbulence, J. Fluid Mech. 421 (2000), 307-338.

[11] A. Cheskidov, D. D. Holm, E. Olson, and E. S. Titi, On a Leray- $\alpha$ model of turbulence, Proc. R. Soc. Lond. Ser. A Math. Phys. Eng. Sci. 461 (2005), 629-649.

[12] P. Constantin and C. Foias, Navier-Stokes equations, Chicago Lectures in Mathematics, University of Chicago Press, Chicago, IL, 1988.

[13] P. Constantin, C. Foias, B. Nicolaenko, and R. Temam, Spectral barriers and inertial manifolds for dissipative partial differential equations, J. Dynam. Differential Equations 1 (1989), 45-73.

[14] M. Coti Zelati and C. G. Gal, Singular limits of Voigt models in fluid dynamics, J. Math. Fluid Mech. 17 (2015), 233-259. 
[15] A. Eden, V. Kalantarov, and S. Zelik. Counterexamples to the regularity of Mane projections in the attractors theory, Russ. Math. Surv. 68 (2013), 199-226.

[16] C. Foias, D. D. Holm, and E. S. Titi, The three dimensional viscous Camassa-Holm equations, and their relation to the Navier-Stokes equations and turbulence theory, J. Dynam. Differential Equations 14 (2002), 1-35.

[17] C. Foias, B. Nicolaenko, G. R. Sell, and R. Temam, Inertial manifolds for the Kuramoto-Sivashinsky equation and an estimate of their lowest dimension, J. Math. Pures Appl. (9) 67 (1988), 197-226.

[18] C. Foias, G. R. Sell, and R. Temam, Inertial manifolds for nonlinear evolutionary equations, J. Differential Equations 73 (1988), 309-353.

[19] C. Foias, G. R. Sell, and E. S. Titi, Exponential tracking and approximation of inertial manifolds for dissipative nonlinear equations, J. Dynam. Differential Equations 1 (1989), 199-244.

[20] B. Fornberg, A numerical study of two-dimensional turbulence, J. Comput. Phys. 25 (1977), 1-31.

[21] C. G. Gal and T. T. Medjo, On a regularized family of models for homogeneous incompressible two-phase flows, J. Nonlinear Science 24 (2014), 1033-1103.

[22] M. Holst, E. Lunasin, and G. Tsogtgerel, Analysis of a general family of regularized Navier-Stokes and MHD models, J. Nonlinear Sci. 20 (2010), 523-567.

[23] V. K. Kalantarov and E. S. Titi, Global attractors and determining modes for the 3D Navier-StokesVoight equations, Chin. Ann. Math. Ser. B 30 (2009), 697-714.

[24] A. Kostianko, Inertial manifolds for the 3D modified-Leray- $\alpha$ model with periodic boundary conditions, J. Dynam. Differential Equations 30 (2018), 1-24.

[25] A. Kostianko and S. Zelik, Inertial manifolds for the 3D Cahn-Hilliard equations with periodic boundary conditions, Commun. Pure Appl. Anal. 14 (2015), 2069-2094.

[26] J. Mallet-Paret and G. R. Sell, Inertial manifolds for reaction diffusion equations in higher space dimensions, J. Amer. Math. Soc. 1 (1988), 805-866.

[27] J. C. McWilliams, The emergence of isolated coherent vortices in turbulent flows, J. Fluid Mech. 146 (1984), 21-43.

[28] M. Miklavcic, A sharp condition for existence of an inertial manifold, J. Dynam. Diff. Eqns 3 (1991), 437-456.

[29] I. Richards, On the gaps between numbers which are sums of two squares, Adv. in Math. 46 (1982), 1-2.

[30] A. Romanov, Sharp estimates for the dimension of inertial manifolds for nonlinear parabolic equations, Izv. Akad. Nauk SSSR Ser. Mat. 43 (1994), 31-47.

[31] A. Romanov, Three counterexamples in the theory of inertial manifolds, Math. Notes 68 (2000), 378-385.

[32] G. R. Sell and Y. You, Inertial manifolds: the non-selfadjoint case, J. Differential Equations 96 (1992), 203-255.

[33] R. Temam, Infinite-dimensional dynamical systems in mechanics and physics, Second edition, Applied Mathematical Sciences, 68. Springer-Verlag, New York, 1997.

[34] J. Vukadinovic, Inertial manifolds for a Smoluchowski equation on the unit sphere, Comm. Math. Phys. 285 (2009), 975-990.

[35] S. Zelik, Inertial manifolds and finite-dimensional reduction for dissipative PDEs, Proc. Roy. Soc. Edinburgh Sect. A 144 (2014), 1245-1327.

E-mail address: cgal@fiu.edu

E-mail address: yanguo@fiu.edu

Department of Mathematics \& Statistics, Florida International University, Miami, Florida 33199, USA

Department of Mathematics \& Statistics, Florida International University, Miami, Florida 33199, USA 